\title{
A Reduced-Order Representation of the Madden-Julian Oscillation Based on Reanalyzed Normal Mode Coherences
}

\author{
VAssili Kitsios And Terence J. O'Kane \\ Oceans and Atmosphere, CSIRO, Hobart, Tasmania, Australia \\ NEDJELJKA ŽAGAR \\ Meteorologisches Institute, Universität Hamburg, Hamburg, Germany
}

(Manuscript received 14 July 2018, in final form 28 April 2019)

\begin{abstract}
The Madden-Julian oscillation (MJO) is presented as a series of interacting Rossby and inertial gravity waves of varying vertical scales and meridional extents. These components are isolated by decomposing reanalysis fields into a set of normal mode functions (NMF), which are orthogonal eigenvectors of the linearized primitive equations on a sphere. The NMFs that demonstrate spatial properties compatible with the MJO are inertial gravity waves of zonal wavenumber $k=1$ and the lowest meridional index $n=0$, and Rossby waves with $(k, n)=(1,1)$. For these horizontal scales, there are multiple small vertical-scale baroclinic modes that have temporal properties indicative of the MJO. On the basis of one such eastward-propagating inertial gravity wave (i.e., a Kelvin wave), composite averages of the Japanese 55-year Reanalysis demonstrate an eastward propagation of the velocity potential, and oscillation of outgoing longwave radiation and precipitation fields over the Maritime Continent, with an MJO-appropriate temporal period. A cross-spectral analysis indicates that only the MJO time scale is coherent between this Kelvin wave and the more energetic modes. Four mode clusters are identified: Kelvin waves of correct phase period and direction, Rossby waves of correct phase period, energetic Kelvin waves of larger vertical scales and meridional extents extending into the extratropics, and energetic Rossby waves of spatial scales similar to that of the energetic Kelvin waves. We propose that within this normal mode framework, nonlinear interactions between the aforementioned mode groups are required to produce an energetic MJO propagating eastward with an intraseasonal phase period. By virtue of the selected mode groups, this theory encompasses both multiscale and tropical-extratropical interactions.
\end{abstract}

\section{Introduction}

The Madden-Julian oscillation (MJO) is an eastwardpropagating intraseasonal mode of variability, with its variance concentrated in the 30-90-day time-scale band. It is a physical phenomenon first characterized by Madden and Julian (1971), involving interactions between tropical deep convection focused within the Maritime Continent, moisture, and atmospheric dynamics (Zhang 2005). Since this initial study there has been a vast array of research into this physical phenomenon, with recent comprehensive reviews in Zhang (2005) and DeMott et al. (2015). In the current study we decompose atmospheric reanalysis into a series of scales using normal mode functions (NMF), and on the basis of these results propose a reduced-order theory of the MJO.

\footnotetext{
Corresponding author: Vassili Kitsios, vassili.kitsios@csiro.au
}

The following literature review hence focuses on the spatiotemporal-scale properties of the MJO and the historical theories explaining its dynamics.

The life cycle of the MJO is typically characterized as being initiated by a strong large-scale deep convective event and associated precipitation over the Maritime Continent. This strong deep convection is linked to surrounding regions of weaker convection via the circulation in the vertical-longitudinal plane extending throughout the troposphere (Madden and Julian 1972; Rui and Wang 1990). In the lower troposphere, at an altitude of approximately $850 \mathrm{hPa}$, the zonal winds converge toward the convective center. In the upper troposphere, at around $200 \mathrm{hPa}$, the zonal winds diverge and travel away from the source of convection. This large-scale convective system propagates eastward and decays in the western Pacific. This first-order view of the MJO suggests a simple baroclinic structure, since the 
anomalous zonal velocity at the upper and lower levels are of opposite sign. In reality, however, this perceived large-scale eastward propagation is due to a hierarchy of smaller spatiotemporal-scale convective systems that on average are initiated eastward of the earlier systems (Nakazawa 1988; Hendon and Salby 1994; Chen et al. 1996). At present this finer vertical-scale structure is less well understood. One of the goals of the current study is to infer a hierarchy of important vertical scales from the NMFs. In doing so we aim to clarify the nonlinear processes that couple westward-propagating Rossby waves, fast eastward inertial gravity waves, and extratropical influences, which together give rise to the intraseasonal eastward propagation of the MJO.

The large-scale horizontal features of the MJO convection were characterized in Wheeler and Kiladis (1999) via the spectral properties of the outgoing longwave radiation (OLR). Here, the meridionally symmetric and antisymmetric components of OLR were spectrally decomposed into longitudinal wavenumber $(k)$ and temporal-frequency $(f)$ components. The MJO was identified as an eastwardpropagating symmetric component of OLR. In the $(k, f)$ plane it has significant variance for wavenumbers $k=1-4$ for time scales longer than 30 days, with $k=1$ having the largest contribution. Faster eastward-propagating Kelvin waves were also identified, which were aligned with solutions of the shallow-water equations of equivalent heights (or depths) between 12 and $50 \mathrm{~m}$. The fast propagation speed (or equivalently short phase period) of Kelvin waves is commonly cited as a reason for why they are not representative of the MJO. Following on from this physical understanding of the MJO, Wheeler and Hendon (2004) devised a real-time multivariate MJO (RMM) index involving the singular value decomposition of OLR, and the longitudinal velocity at 200 and $850 \mathrm{hPa}$, all meridionally averaged within the region $15^{\circ} \mathrm{S}-15^{\circ} \mathrm{N}$. The first two principal components (referred to as RMM1 and RMM2) are shown to have dominant variance within the intraseasonal time-scale band.

Theories of the MJO typically involve prescribed relationships between convection, moisture, and atmospheric dynamics. Initial models were developed on the basis of wave-conditional instability of the second kind (CISK) theory, in which Kelvin waves become unstable because of low-level convective heating (Lindzen 1974; Lau and Peng 1987; Chang and Lim 1988). These models, however, did not predict the appropriate longitudinal scale of the MJO. The wind-induced surface heat exchange (WISHE) theory of Emanuel (1987) proposed eastward-propagating tropical waves were perturbed via enhanced evaporation. The WISHE model produces modes of appropriate longitudinal scale, and propagation speed, but with inconsistent perturbation structure during the Northern Hemisphere winter. In the frictional wave-CISK theory equatorial Rossby waves are also included, the interaction of which produces MJO-like properties of appropriate length and time scales (Wang and Rui 1990; Wang and Li 1994).

Another prevailing theory of the MJO is that it is driven by a moisture mode instability. This type of instability depends on the evolution of the humidity field, with its growth governed by feedbacks that further moisten the atmosphere (Neelin and Yu 1994; Fuchs and Raymond 2005). Raymond and Fuchs (2009) identify that in general this moistening process occurs in the presence of negative gross moist stabilities (GMSs). GMS is a vertically integrated measure of the moist static stability in the atmosphere (Neelin and Held 1987). Sobel and Maloney (2012) also find in their idealized model of the MJO that eastward-propagating waves can only be growing if the effective GMS is less than zero.

Majda and Stechmann (2009) developed a minimal dynamical model of the MJO capturing the interactions between lower-tropospheric moisture and convectively coupled wave dynamics of prescribed meridional and baroclinic structure. Within this framework they identify a mechanism for fast Kelvin waves and westerly propagating equatorial Rossby waves to couple to each other and become dispersive. This model produced an MJO with a small group velocity, intraseasonal phase speed, and a horizontal quadrapole vortex structure. Chen et al. (2016) extended on this work to model both the onset and decay of the MJO via interactions with extratropical and barotropic modes.

In Frederiksen and Lin (2013) the MJO is characterized by a coupled tropical-extratropical mode energized by moist baroclinic-barotropic instability within a threedimensional basic state. The linear primitive-equation instability model of Frederiksen (2002) is used to simulate eternal-January basic states. Within this framework the MJO is a single mode of dominant zonal wavenumber $k=1$, first internal baroclinic structure in the tropics, equivalent barotropic structure in the extratropics, and a phase period of 34.4 days (Frederiksen and Lin 2013). Other intraseasonal modes have also been identified using this approach with phase periods between 28 and 60 days (Frederiksen and Frederiksen 1993; Frederiksen 2002; Frederiksen and Lin 2013).

There are multiple theories that arrive at length and time scales consistent with the MJO, each of which adopts different approaches and hence has an alternate explanation of the nature of the MJO. At present there is no consensus on the appropriate framework, nor as to which minimal set of scales are required to reproduce the MJO dynamics. Yano and Tribbia (2017) also make the point that the results of certain successful theories 
are sensitive to parameter selection. Here we divorce ourselves from any parameter selection issues by processing three-dimensional global reanalysis data to identify which atmospheric scales have the appropriate spatiotemporal MJO properties, and the observed relationships between them.

Žagar and Franzke (2015) undertook a scale decomposition of the MJO present in ERA-Interim on the basis of NMFs. NMFs are the eigenvectors of the linearized primitive equations on a sphere, simultaneously capturing the variability in the horizontal velocity field and geopotential height, with the eigenvalues being the temporal period of the mode. The NMFs are characterized by a zonal wavenumber $k$, meridional wavenumber $n$, and vertical mode number $m$, which are in turn decomposed into Rossby waves and westerly and easterly traveling inertial gravity waves. Žagar and Franzke (2015) calculated the MJO-associated spectrum of NMF variance using with the RMM index. They found the largest percentage of the global variance is in the $n=1$ Rossby mode of baroclinic structure with vertical modes $m>3$. Using a similar approach Castanheira and Marques (2015) isolated the MJO from ERA-Interim via a crossspectral analysis between the NMF decomposed $k=1$ and $n=1$ Rossby wave, and the OLR field. However, in isolation (i.e., not interacting with any other modes nor itself), Rossby waves travel westward. In addition, the eigenvalues associated with this mode indicates that the phase periods are too short to be that of the MJO. While the dominant projection of the MJO is onto this particular mode, the dynamics cannot be described by it alone. However, we propose that the pertinent dynamics can be described via its interaction with other NMFs.

In the present paper we extend the previous NMF applications and show that there are smaller-scale vertical modes that do have the appropriate phase periods, and other physical properties representative of the MJO. Unlike the Žagar and Franzke (2015) and Castanheira and Marques (2015) studies, the approach presented here does not require the RMM index nor the OLR field to isolate the MJO. We do, however, compare our results to the RMM index and also generate composites of the OLR field as means of validation. The NMF decomposition presented within is not intended to be a real-time index of the MJO as in Wheeler and Hendon (2004). Instead the intention is to use the NMFs to further understand the three-dimensional nature of the MJO, and its multiscale interactions.

The manuscript is organized as follows. In section 2 we outline the NMF theory, with the NMFs themselves calculated in section 3. Candidate NMFs representative of the MJO are determined on the basis of their temporal and spatial properties. The NMFs are then projected onto the daily fields of the Japanese 55-year Reanalysis (JRA-55; Kobayashi et al. 2015) with the spectral decomposition of the variance presented in section 4. A cross-spectral analysis is undertaken in section 5 to infer the potential for interactions between the pertinent modes. In section 6 , one of the MJO-like NMF modes is used to produce three-dimensional phase averages. This same mode is then used to composite large and persistent MJO events in section 7. This analysis demonstrates the propagation of the velocity potential, and oscillation of the OLR and precipitation fields with the appropriate intraseasonal period. The temporal evolution of a combination of NMF modes are shown to be highly correlated with the RMM index in section 8. Finally, concluding remarks are made in section 9 .

\section{Normal mode theory}

NMFs decompose the flow by vertical scale via vertical structure functions (VSFs), and horizontal scale and mode type via horizontal structure functions (HSFs). The linearized equations of motion from which the NMFs are derived are described below, followed by the two eigenvalue problems (EVPs) required to calculate the VSFs and HSFs. As presented in detail in Kasahara and Puri (1981) and Žagar et al. (2015), the NMFs are derived from the linearized hydrostatic baroclinic primitive equations of the atmosphere on a sphere. The equations are given by

$$
\begin{aligned}
\frac{\partial u^{\prime}}{\partial t}-2 \Omega \sin (\phi) v^{\prime} & =-\frac{g}{a \cos \phi} \frac{\partial h^{\prime}}{\partial \lambda}, \\
\frac{\partial v^{\prime}}{\partial t}+2 \Omega \sin (\phi) u^{\prime} & =-\frac{g}{a} \frac{\partial h^{\prime}}{\partial \phi}, \quad \text { and } \\
\frac{\partial}{\partial t}\left\{\frac{\partial}{\partial \sigma}\left[\frac{g \sigma}{R \Gamma_{0}(\sigma)} \frac{\partial h^{\prime}}{\partial \sigma}\right]\right\} & =\nabla \cdot \mathbf{V}^{\prime},
\end{aligned}
$$

where $g=9.81 \mathrm{~m} \mathrm{~s}^{-2}$ is gravity, $t$ is time, $\lambda$ is longitude, $\phi$ is latitude, and the vertical coordinate $\sigma=p / p_{s}$, where $p$ is the pressure and $p_{s}$ the surface pressure. These equations govern the linear evolution of the state vector $\left[u^{\prime}, v^{\prime}, h^{\prime}\right]$ in a zero-flow climate, where $u^{\prime}$ and $v^{\prime}$ are the longitudinal and meridional velocity components, respectively. The modified geopotential height $h^{\prime}=z+R T_{0}(\sigma) \ln \left(p_{s}\right) / g$, where $z$ is the standard geopotential height, $T_{0}(\sigma)$ is the horizontally averaged temperature at a given $\sigma$ level, and $R=287 \mathrm{~J} \mathrm{~kg}^{-1} \mathrm{~K}^{-1}$ is the gas constant in air. Note, $z$ is a function of both temperature and specific humidity. The static stability profile $\Gamma_{0}(\sigma)$ is given by 


$$
\Gamma_{0}(\sigma)=\frac{R T_{0}(\sigma)}{c_{p} \sigma}-\frac{d T_{0}(\sigma)}{d \sigma}
$$

where $c_{p}=1004.6 \mathrm{~J} \mathrm{~kg}^{-1} \mathrm{~K}^{-1}$ is the specific heat of air at constant pressure. Equations (1)-(3) are augmented with the surface boundary condition of

$$
\frac{\partial h^{\prime}}{\partial \sigma}+\frac{\Gamma_{0}}{T_{0}} h^{\prime}=0
$$

at $\sigma=1$, and the top boundary condition of

$$
\sigma \frac{\partial h^{\prime}}{\partial \sigma}=0
$$

at $\sigma=0$.

The vertical and horizontal variations of the state vector are assumed separable according to

$$
\left[u^{\prime}, v^{\prime}, h^{\prime}\right]^{\mathrm{T}}(\lambda, \phi, \sigma, t)=[u, v, h]^{\mathrm{T}}(\lambda, \phi, t) \times G(\sigma),
$$

where $G(\sigma)$ is a function of only the vertical coordinate. Substituting (7) into (1)-(3) returns two EVPs. The first is that of the VSF given by

$$
\frac{d}{d \sigma}\left[\frac{\sigma}{S} \frac{d G(\sigma)}{d \sigma}\right]=-\frac{H_{\star}}{D} G(\sigma),
$$

where $S(\sigma)=R \Gamma_{0}(\sigma) /\left(g H_{\star}\right)$, and $H_{\star}=8 \mathrm{~km}$ is a scaling factor. We use the notation $G_{m}(\sigma)$ to refer to the $m$ th VSF (i.e., eigenvector), and $D_{m}$ the associated equivalent height (i.e., eigenvalue). Using the decomposition in (7), the surface boundary condition in (5) applied at $\sigma=1$ becomes

$$
\frac{d G(\sigma)}{d \sigma}+\frac{\Gamma_{0}}{T_{0}} G(\sigma)=0
$$

The top boundary condition becomes

$$
\sigma \frac{d G(\sigma)}{d \sigma}=0,
$$

which is applied at some small value of $\sigma=\sigma_{T}$. This eigenvalue problem has the property that all eigenvalues (i.e., equivalent heights) are positive. Note that this boundary condition is applied at $\sigma_{T}$ as opposed to precisely zero, as a means of avoiding the issue of the VSF EVP becoming singular. This means that we are not solving the exact analytical form of the equations presented in (8)-(10). However, Žagar et al. (2009) demonstrates that small differences in the location of the model top for the given vertical discretization and static stability profile have no significant impact on the VSFs.
The second EVP is that of the HSF that require $D_{m}$ as an input. It is given by

$$
\frac{\partial}{\partial \tilde{t}} \mathbf{W}_{m}+\mathbf{L}_{m} \mathbf{W}_{m}=0
$$

where $\mathbf{W}_{m}(\lambda, \phi, \tilde{t})=\left(\tilde{u}_{m}, \tilde{\boldsymbol{v}}_{m}, \tilde{h}_{m}\right)^{\mathrm{T}}$, with nondimensional quantities $\tilde{u}_{m}=u_{m} / \sqrt{g D_{m}}, \tilde{v}_{m}=v_{m} / \sqrt{g D_{m}}, \tilde{h}_{m}=h_{m} / D_{m}$, and $\tilde{t}=2 \Omega t$. The linear operator $\mathbf{L}_{m}$ is given by

$$
\mathbf{L}_{m}=\gamma_{m}\left[\begin{array}{ccc}
0 & -\sin (\phi) / \gamma_{m} & \frac{1}{\cos \phi} \frac{\partial}{\partial \lambda} \\
\sin (\phi) / \gamma_{m} & 0 & \frac{\partial}{\partial \phi} \\
\frac{1}{\cos \phi} \frac{\partial}{\partial \lambda} & -\tan \phi+\frac{\partial}{\partial \phi} & 0
\end{array}\right],
$$

where $\gamma_{m}=\sqrt{g D_{m}} /(2 a \Omega)$. The solutions to (11) take the form

$$
\mathbf{W}_{m}(\lambda, \phi, \tilde{t})=\mathbf{H}_{k n m}^{q}(\lambda, \phi) e^{-i v_{k n m}^{q} \tilde{t}},
$$

where $\mathbf{H}_{k n m}^{q}(\lambda, \phi)$ represent the HSFs (i.e., eigenvectors) with zonal wavenumber $k$, meridional index $n$, vertical index $m$, and mode type $q$. The associated dimensionless frequency (i.e., eigenvalue) is $\nu_{k n m}^{q}$. Separation of variables about the periodic longitudinal direction produces

$$
\mathbf{H}_{k n m}^{q}(\lambda, \phi)=\boldsymbol{\Theta}_{k n m}^{q}(\phi) e^{i k \lambda},
$$

where $\Theta_{k n m}^{q}(\phi)=\left[U_{k n m}^{q}(\phi),-i V_{k n m}^{q}(\phi), Z_{k n m}^{q}(\phi)\right]$, with $U_{k n m}^{q}(\phi), V_{k n m}^{q}(\phi)$, and $Z_{k n m}^{q}(\phi)$ meridionally dependent profiles of the zonal velocity, meridional velocity, and modified geopotential heights, respectively.

Since there are three components in the state vector, there are three eigensolutions (or mode types, $q$ ) per scale triplet $(k, n, m)$. One of the solutions is a lowfrequency Rossby wave-like balanced (BAL) mode. For even $n, Z_{k n m}^{q}$ and $U_{k n m}^{q}$ are antisymmetric about the equator, while $V_{k n m}^{q}$ is symmetric. For odd $n$, the symmetry properties are swapped. The remaining two solutions are high-frequency inertial gravity (IG) waves, with symmetry properties swapped in comparison to the BAL modes. One solution is an eastward-propagating IG wave (EIG), and the other a westward-propagating IG wave (WIG). We use the index $q$ of values $(1,2,3)$, to refer to the mode types (EIG, WIG, BAL). Throughout the manuscript we use the subscript $\left(k, n, D_{m}\right)$ to refer to the horizontal and vertical structure of a given mode class. For example the EIG mode of indices $(k, n, m)=(1,0,28)$, is denoted by $\operatorname{EIG}_{\left(k, n, D_{m}\right)}=$ $\mathrm{EIG}_{\left(1,0, D_{28}\right)}=\mathrm{EIG}_{(1,0,7 \mathrm{~m})}$. 

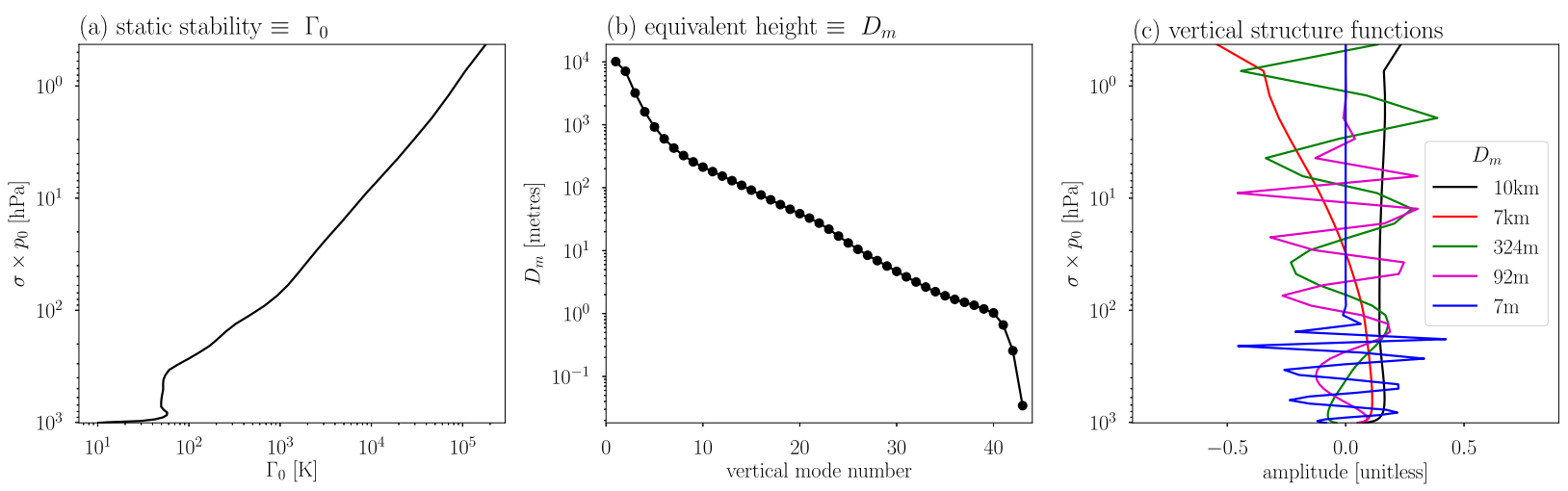

FIG. 1. Vertical structure function eigensolution: (a) static stability profile vs an indicative pressure $\sigma \times p_{0}$, where $p_{0}=1013 \mathrm{hPa}$; (b) equivalent height $D_{m}$, or eigenvalues of the VSF EVP; and (c) a subset of vertical modes, or eigenvectors of the VSF EVP.

\section{Normal mode functions of the Japanese 55-year Reanalysis}

The VSF and HSF modes derived in the previous section are calculated here using the MODES code developed and described in Žagar et al. (2015). Upon defining the vertical discretization on the terrain-following coordinate levels $\sigma$, the only input data required for the calculation of the VSF modes are the static stability profiles $\Gamma_{0}(\sigma)$. The $\sigma$ grid is defined such that for a nominal surface pressure of $1013 \mathrm{hPa}$, there are 7 levels below $800 \mathrm{hPa}, 6$ levels between 800 and $500 \mathrm{hPa}$, 13 levels from 500 to $100 \mathrm{hPa}, 9$ levels between 100 and $10 \mathrm{hPa}$, and 8 levels from 10 to $0.5 \mathrm{hPa}$, for a total of 43 levels.

Daily temperature, wind and moisture fields of JRA-55 from 1 January 1958 to 31 December 2016 are interpolated to the above-defined $\sigma$ levels. JRA-55 temperature fields are first formed using 37 isobaric levels defined from 1 to $1000 \mathrm{hPa}$, and augmented by the surface temperature. These fields are then interpolated onto the above-defined $\sigma$ grid, and averaged across each $\sigma$ level to produce $T_{0}(\sigma, t)$. The daily static stability profiles $\Gamma_{0}(\sigma, t)$ are then calculated according to (4). The $\Gamma_{0}(\sigma, t)$ profiles are then time averaged to produce $\Gamma_{0}(\sigma)$, which is illustrated in Fig. 1a. This profile is everywhere positive and hence stable.

The eigenvalues of the VSF EVP in (8) are the equivalent heights $D_{m}$ and illustrated in Fig. 1b. The equivalent heights decrease monotonically with vertical mode index $m$. The $m$ th VSF has $m-1$ zero crossings. The $m=1$ mode has no zero crossings and is hence referred to as the barotropic mode, here with an equivalent height of $D_{1}=10 \mathrm{~km}$. The $m=2$ mode has one crossing and consequently referred to as the first baroclinic mode, of equivalent height $D_{2}=7 \mathrm{~km}$. These modes are illustrated in Fig. 1c, along with the $m=8, m=15$, and $m=28 \mathrm{VSF}$, with respective equivalent heights of
$D_{8}=324 \mathrm{~m}, D_{15}=92 \mathrm{~m}$, and $D_{28}=7 \mathrm{~m}$. As the equivalent height decreases (or equivalently $m$ increases) the structures of the VSFs are also concentrated closer to the surface.

The HSF EVP requires $D_{m}$ as an input, and hence each vertical mode $m$ has a unique set of HSFs and associated eigenvalues $\nu_{k n m}^{q}$. HSF EVP solutions are calculated for $D_{m}>1 \mathrm{~m}$. The periods $\left(1 / \nu_{k n m}^{q}\right)$ calculated from the HSF EVP are illustrated in Fig. 2a for the vertical mode with an equivalent height of $7 \mathrm{~m}$. We select this equivalent height since the EIG mode with $k=1$ and $n=0$ has a time scale central to the intraseasonal band of 56 days. The EIG modes (blue dots) are the only easterly propagating modes, having positive $k$. Both the WIG (red dots) and BAL (black dots) modes have a negative $k$ and hence propagate westerly. The bold dots represent the $n=1$ meridional index for each of these mode types. The separation in time scales between the IG and BAL modes also decreases as the equivalent heights decrease, or equivalently as $m$ increases (Žagar et al. 2015). As $n$ increases, the time scale of the IG modes decrease, while that of the BAL modes increase. Two other subclasses are also explicitly illustrated. The mixed Rossby-gravity (MRG) modes are by definition the BAL modes with $n=0$ (for all $k$ and $m$ ). Likewise the Kelvin wave are the EIG modes with $n=0$. Kelvin waves have the particular property that their phase speed $\left(\nu_{k n m}^{q} / k\right)$ is equivalent to their group velocity $\left(\partial \nu_{k m n}^{q} / \partial k\right)$.

At this stage we look to identify a set of modes representative of the MJO. To restate, the MJO is an eastward-propagating tropics centric intraseasonal oscillation with horizontal velocity components of dominant zonal wavenumber $k=1$, and whose zonal velocity is also predominantly symmetric about the equator. For the inertial gravity waves, the $n=0$ modes are tropics centric with their magnitude decreasing monotonically 

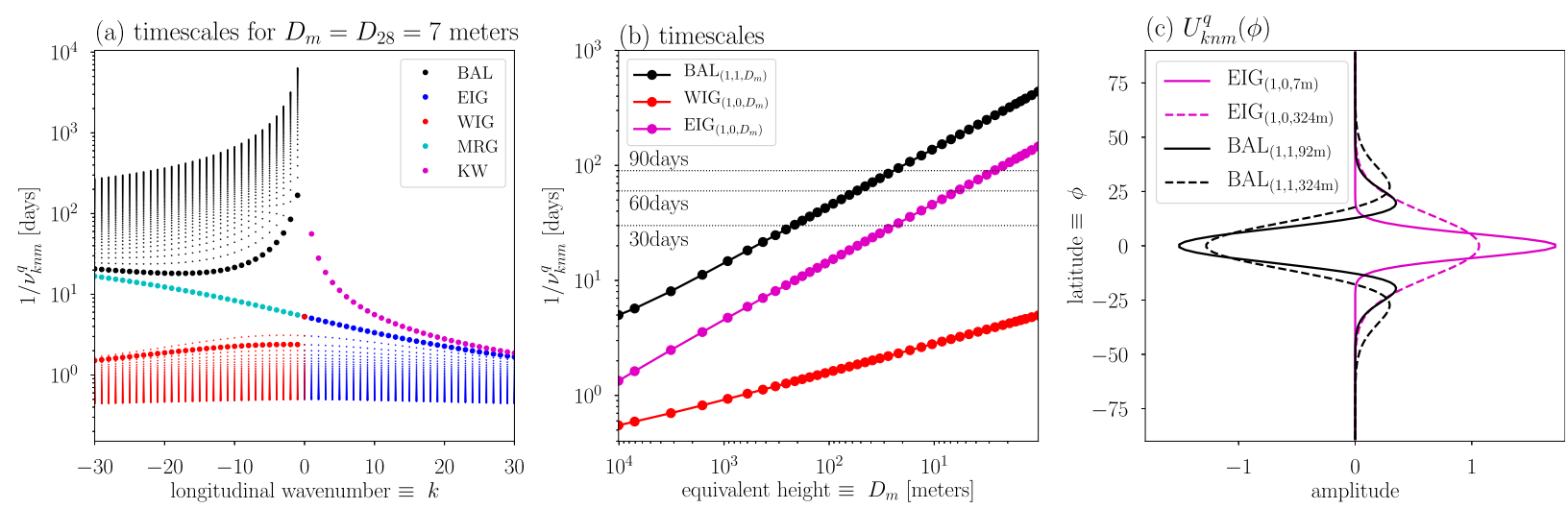

FIG. 2. Horizontal structure function eigensolutions: (a) time scales $1 / \nu_{k n m}^{q}$ of the HSF for vertical mode of equivalent height $=7 \mathrm{~m}$ for balanced (BAL; large dots: $n=1$; small dots: $n>1$ ), easterly propagating inertial gravity waves (EIG; large dots: $n=1$; small dots: $n>1$ ), westerly propagating inertial gravity waves (WIG; large dots: $n=1$; small dots for all other $n$ ), mixed Rossby-gravity (MRG; equivalent to BAL: $n=0)$ and Kelvin waves (KW; equivalent to EIG: $n=0)$; (b) time scales $1 / \nu_{k n m}^{q}$ of the HSF for EIG with $(k, n)=(1,0)$, WIG with $(k, n)=(1,0)$, and BAL with $(k, n)=(1,1)$ vs the equivalent height of each vertical mode; and (c) the longitudinal velocity component $U_{k n m}^{q}(\phi)$ of the horizontal structure functions for EIG modes with $\left(k, n, D_{m}\right)=(1,0,7 \mathrm{~m})$ and $(1,0,324 \mathrm{~m})$, and BAL modes with $\left(k, n, D_{m}\right)=(1,1,92 \mathrm{~m})$ and $(1,1,324 \mathrm{~m})$

with distance from the equator, and also have the appropriate symmetry properties. Hence the horizontalscale pair of $(k, n)=(1,0)$ is an appropriate starting point for the EIG and WIG modes. For the Rossby waves, the $n=1$ modes have the appropriate symmetry properties, hence the $(k, n)=(1,1)$ is the appropriate horizontal-scale pair. The time scales of the associated Kelvin wave (EIG with $n=0$ ), WIG and BAL modes are illustrated as a function $D_{m}$ in Fig. 2 b. This figure demonstrates power-law relationships between the time scales and equivalent depths. Both the Kelvin wave and BAL modes contains temporal scales within the intraseasonal time-scale band. Specifically the $\mathrm{EIG}_{\left(1,0, D_{m}\right)}$ modes, or Kelvin waves, with $D_{m}$ of order $10 \mathrm{~m}$ have time scales between 30 and 90 days. As mentioned above, the eigenvalue associated with $\mathrm{EIG}_{(1,0,7 \mathrm{~m})}$ has a time scale of 56 days and is central to this intraseasonal range. The balanced modes, $\mathrm{BAL}_{\left(1,1, D_{m}\right)}$, have phase periods within intraseasonal time-scale band for $D_{m}$ of order $100 \mathrm{~m}$, with $\operatorname{BAL}_{(1,1,92 \mathrm{~m})}$ having a time scale of 46 days. The linear dispersion relationships are such that the Kelvin waves propagate eastward, while the BAL modes propagate westward. In order for the BAL modes to propagate eastward like the MJO (as later observed in the reanalysis), we hypothesize that some form of interaction with these modes is required to modify their effective dispersion relations.

The abovementioned NMFs have a meridional structure appropriate for the MJO as indicated by the zonal velocity component of the HSFs. Four HSFs are illustrated in Fig. 2c: the Kelvin wave with a 56-day phase period, $\operatorname{EIG}_{(1,0,7 \mathrm{~m})}$; the most energetic Kelvin wave, $\mathrm{EIG}_{(1,0,324 \mathrm{~m})}$; the Rossby wave with a 46-day phase period, $\mathrm{BAL}_{(1,1,92 \mathrm{~m})}$; and an energetic Rossby

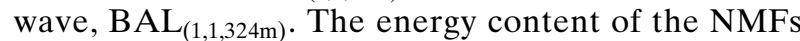
are discussed in the following section. The zonal velocity of the abovementioned modes are tropics centric with their maximum magnitude located at the equator, and are also symmetric about the equator. Note, since these profiles are the eigenvectors of the HSF EVP, the sign of the modes is arbitrary. The EIG $_{(1,0,7 \mathrm{~m})}$ mode has significant amplitudes between $15^{\circ} \mathrm{S}$ and $15^{\circ} \mathrm{N}$. The $\mathrm{EIG}_{(1,0,324 \mathrm{~m})}$ mode is far broader, and is nonzero within extratropics from $50^{\circ} \mathrm{S}$ to $50^{\circ} \mathrm{N}$. The $\mathrm{BAL}_{(1,1,92 \mathrm{~m})}$ mode changes sign at $10^{\circ}$ either side of the equator, with secondary peaks at $15^{\circ} \mathrm{S}$ and $15^{\circ} \mathrm{N}$. The $\mathrm{BAL}_{(1,1,324 \mathrm{~m})}$ mode has the same topological features as $\operatorname{BAL}_{(1,1,92 \mathrm{~m})}$, but is broader and extends farther into the extratropics. In general, the inertial gravity waves with $n=0$ and BAL modes with $n=1$ become more equatorially trapped as $m$ increases as well as with increasing $k$.

\section{Scale and mode type decomposition}

The contribution of the VSFs and HSFs to the daily JRA-55 fields are calculated using the NMF orthogonality properties presented in Žagar et al. (2015). Physical space fields are decomposed according to

$$
\left[\begin{array}{l}
u(\lambda, \phi, \sigma, t) \\
v(\lambda, \phi, \sigma, t) \\
h(\lambda, \phi, \sigma, t)
\end{array}\right]=\sum_{m=1}^{M} \mathbf{S}_{m} G_{m}(\sigma) \mathbf{X}_{m}(\lambda, \phi, t) .
$$



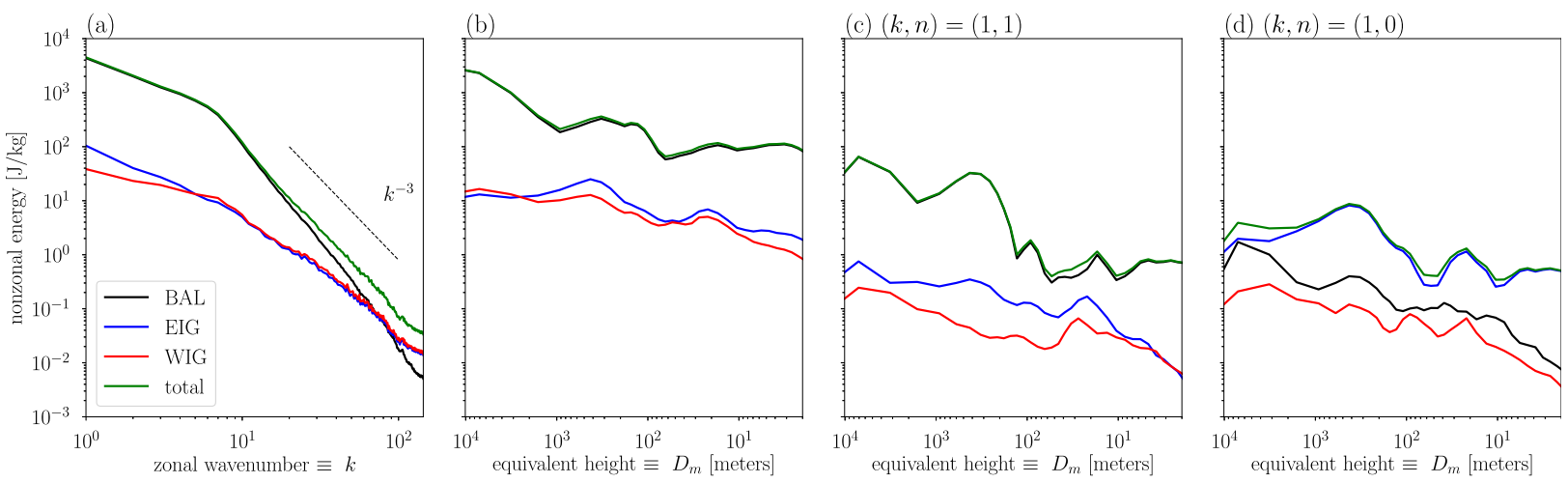

FIG. 3. Time-averaged spectral contribution from each of the NMFs: (a) zonal wavenumber spectra of total energy (i.e., potential and kinetic) summed over $n$ and $m$ decomposed into EIG, WIG, and BAL components, with the dashed black line representing the $k^{-3}$ decay rate; (b) vertical mode spectra of total nonzonal energy summed over $k$ and $n$ as a function of equivalent height; (c) as in (b), but for only the $(k, n)=(1,1)$ horizontal mode pair; and (d) as in (b), but for only the $(k, n)=(1,0)$ horizontal mode pair. Legend and vertical scale in (a) is applicable to all plots.

The total number of vertical modes is $M$, and

$$
\mathbf{S}_{m}=\left[\begin{array}{ccc}
\sqrt{g D_{m}} & 0 & 0 \\
0 & \sqrt{g D_{m}} & 0 \\
0 & 0 & D_{m}
\end{array}\right]
$$

The dimensionless horizontal coefficients vector is given by

$$
\mathbf{X}_{m}(\lambda, \phi, t)=\sum_{k=-K}^{K} \sum_{n=0}^{N} \sum_{q=1}^{3} \chi_{k n m}^{q}(t) \mathbf{H}_{k n m}^{q}(\lambda, \phi)
$$

where $K$ is the zonal truncation wavenumber, $N$ is the meridional truncation wavenumber, and $q$ takes on the values $(1,2,3)$, referring to mode types (EIG, WIG, BAL). Note, this notation is different from Žagar et al. (2015) with $N$ one-third of the size, because of the introduction of index $q$ to explicitly represent the BAL, EIG, and WIG motions in the summation. The scalar complex expansion coefficients $\chi_{k n m}^{q}$ are then calculated according to

$$
\chi_{k n m}^{q}(t)=\frac{1}{2 \pi} \int_{0}^{2 \pi} \int_{-1}^{1} \mathbf{X}_{m}(\lambda, \phi, t)\left[\mathbf{H}_{k n m}^{q}\right]^{*} d \mu d \lambda,
$$

where $\mu=\sin \phi$ and the asterisk is the complex conjugate operation. The energy of mode type $q$ for a given scale triplet is $E_{k n m}^{q}=\left\langle\chi_{k n m}^{q}(t) \chi_{k n m}^{q}(t)^{*}\right\rangle D_{m} g / 2$, where the angle brackets denote time averaging. The energy spectra of mode type $q$, as a function of a particular index (e.g., $k$ ), is given by the sum of $E_{k n m}^{q}$ over the remaining indices (e.g., $n$ and $m$ ). The total energy is the sum of energy from all mode classes.

The expansion coefficients are calculated for the daily JRA-55 fields from 1 January 1958 to 31 December 2016. All of the analysis throughout the paper is undertaken over this period. The time-averaged zonal wavenumber spectrum of each mode type and the total energy is illustrated in Fig. 3a. The total energy exhibits a $k^{-3}$ decay consistent with the constant enstrophy flux inertial range present in two-dimensional turbulence. When broken down into its components the BAL spectra is steeper than both EIG and WIG. The BAL spectra is the dominant component in the large scales (small wavenumbers). The IG spectra, given by the sum of EIG and WIG spectra, has more energy from $k \approx 40$ onward.

The wave energy (i.e., excluding the $k=0$ component) in each vertical mode is illustrated in Fig. 3b, and indicates that the BAL component is dominant for all equivalent heights. Of these, the barotropic mode $\left(D_{m}=D_{1}=10 \mathrm{~km}\right)$ is the most energetic. The BAL modes are again dominant when considering only the $(k, n)=(1,1)$ horizontal-scale pair, as illustrated in Fig. 3c. The first baroclinic mode $\left(D_{m}=D_{2}=7 \mathrm{~km}\right)$ has the greatest energy contribution for $k=1$ balanced modes, with a secondary peak centered at $D_{m}=324 \mathrm{~m}$. The latter is the $\mathrm{BAL}_{(1,1,324 \mathrm{~m})}$ mode discussed in section 3. The $\operatorname{BAL}_{(1,1,92 \mathrm{~m})}$ mode with an eigenvaluedetermined time scale central to the intraseasonal band has an order of magnitude less energy. When considering only the $(k, n)=(1,0)$ horizontal-scale pair the EIG component is now dominant for all $m$, as illustrated in Fig. 3d. The most energy occurs at $D_{m}=324 \mathrm{~m}$, which is associated with the $\mathrm{EIG}_{(1,0,324 \mathrm{~m})}$ mode discussed in the previous section. The $\mathrm{EIG}_{(1,0,7 \mathrm{~m})}$ mode, with a time scale of 56 days, has an order of magnitude less energy as compared to $\mathrm{EIG}_{(1,0,324 \mathrm{~m})}$.

\section{Cross-spectral analysis}

From the analysis in section $3, \mathrm{EIG}_{(1,0,7 \mathrm{~m})}$ is the only mode with an MJO-like horizontal structure, eastward 

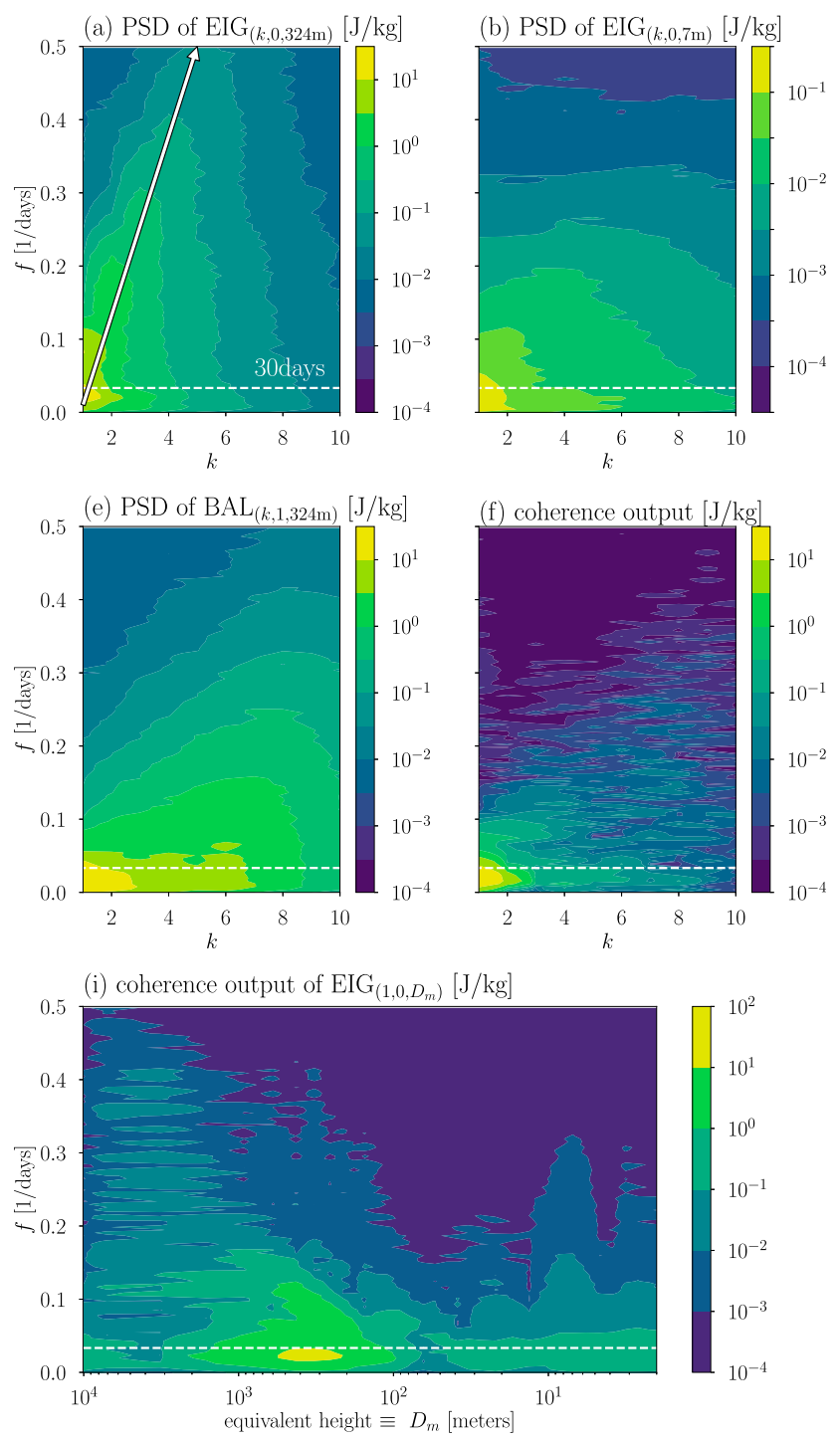
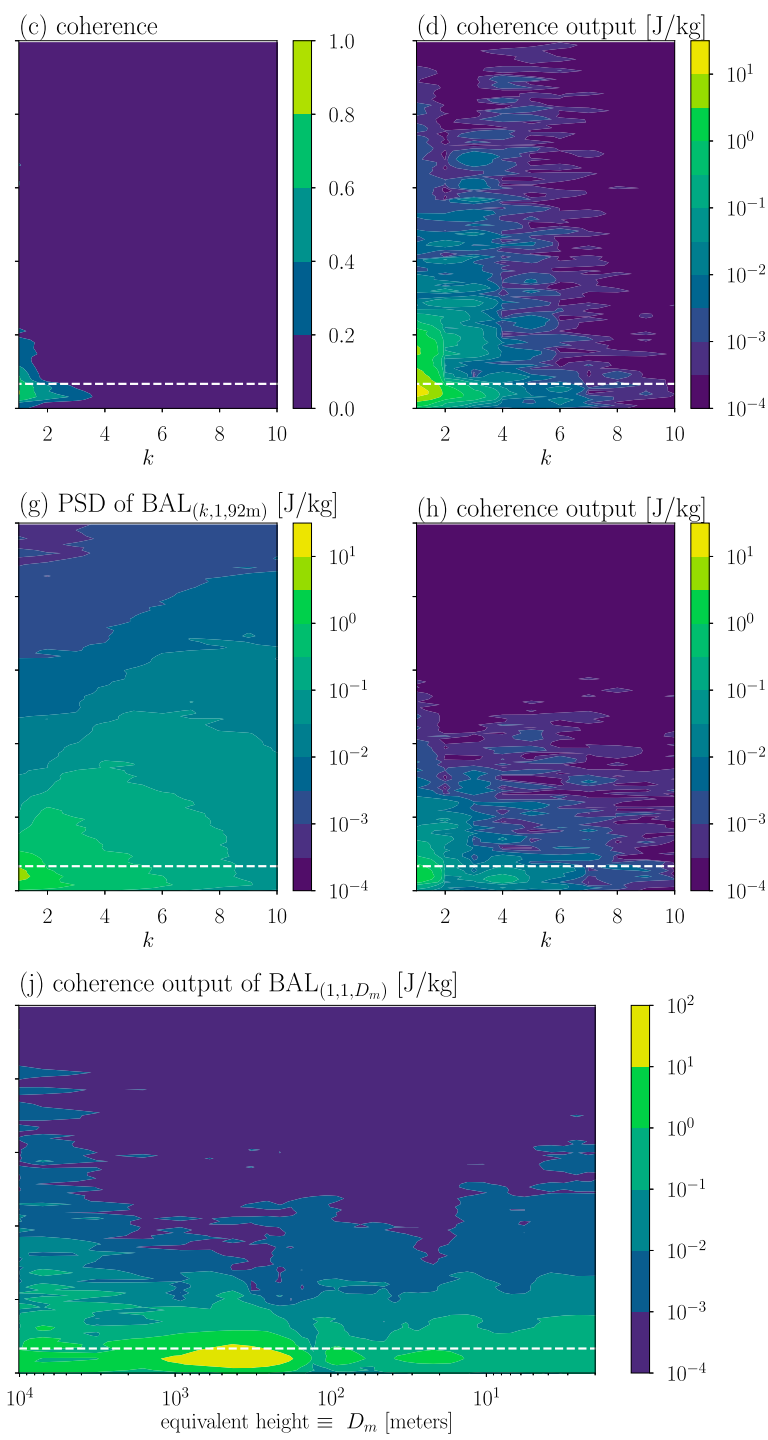

FIG. 4. Spectral coherence properties of the NMFs: (a) power spectral density in the zonal wavenumber $(k)$-frequency $(f)$ plane of $\mathrm{EIG}_{(k, 0,324 \mathrm{~m})}$, with the white arrow indicating the fast Kelvin wave component; (b) as in (a), but for EIG $(k, 0,7 \mathrm{~m})$; (c) coherence (squared correlation in spectral space) in the $(k, f)$ plane between $\operatorname{EIG}_{(k, 0,324 \mathrm{~m})}$ and $\mathrm{EIG}_{(1,0,7 \mathrm{~m})} ;(\mathrm{d})$ coherence output (correlated variance in spectral space) in the $(k, f)$ plane of $\mathrm{EIG}_{(k, 0,324 \mathrm{~m})}$ with respect to $\mathrm{EIG}_{(1,0,7 \mathrm{~m})} ;(\mathrm{e})$ power spectral density of $\mathrm{BAL}_{(k, 1,324 \mathrm{~m})}$; (f) coherence output of $\operatorname{BAL}_{(k, 1,8)}$ with respect to $\mathrm{EIG}_{(1,0,7 \mathrm{~m})}$; $(\mathrm{g})$ power spectral density of $\mathrm{BAL}_{(k, 1,92 \mathrm{~m})}$; (h) coherence output of $\mathrm{BAL}_{(k, 1,92 \mathrm{~m})}$ with respect to $\mathrm{EIG}_{(1,0,7 \mathrm{~m})}$; (i) coherence output of $\mathrm{EIG}_{(1,0, m)}$ with respect to $\mathrm{EIG}_{(1,0,7 \mathrm{~m})}$ for all vertical modes represented by their equivalent height; and (j) coherence output of $\operatorname{BAL}_{(1,1, m)}$ with respect to $\mathrm{EIG}_{(1,0,7 \mathrm{~m})}$ for all vertical modes. Vertical axes in (a), (e), and (i) are applicable to all plots. The horizontal dashed vertical line in all plots indicates a time scale of 30 days.

propagation, and an eigenvalue-determined phase period central to the intraseasonal time-scale band. However, as demonstrated in section 4 this particular mode does not have a dominant contribution to the energy. Of the $\mathrm{EIG}_{\left(1,0, D_{m}\right)}$ modes, $D_{m}=324 \mathrm{~m}$ is the most energetic, but has an eigenvalue-derived phase period too short to be associated with the MJO. The spectral structure of energy contained within these scales of EIG modes is illustrated via the variance distribution in the $(k, f)$ plane, where $f$ is the temporal frequency. Fourier transforms are calculated for the expansion coefficient $\left[\chi_{k n m}^{q}(t)\right]$ anomalies about the yearly cycle, scaled by $\sqrt{D_{m} g / 2}$ to represent the energy content. The power spectral density (PSD) of the EIG modes with $n=0, D_{m}=324 \mathrm{~m}$, and $1 \leq k \leq 10$ is illustrated in Fig. 4a. Note that only the positive frequencies are visualized. It indicates that there are in fact two main propagation speeds: one fast and one slow. The fast component is associated with the classical view of a Kelvin wave and is indicated by the white arrow 
overlaid in the contour plot. The slow component is concentrated within time scales longer than 30 days, as indicated by the white horizontal dashed line, and is presumably associated with the MJO. These features are topologically consistent with the OLR spectra in Wheeler and Kiladis (1999). The PSD of the EIG mode with $n=0$ and $D_{m}=7 \mathrm{~m}$ is illustrated in Fig. $4 \mathrm{~b}$, and indicates that the slow component is dominant.

The covariability and potential for interaction between $\mathrm{EIG}_{(1,0,7 \mathrm{~m})}$ with the correct phase period, and the $\mathrm{EIG}_{(k, 0,324 \mathrm{~m})}$ high energy modes, is determined via their spectral coherence. The magnitude-squared coherence (referred to simply as coherence from this point onward) is given by the squared magnitude of the cross-spectral density between two complex time series divided by the PSDs of both time series. Figure $4 \mathrm{c}$ indicates that only the slow component of these modes are highly coherent. The coherence output in Fig. $4 \mathrm{~d}$ is defined as the coherence in Fig. 4c multiplied by the PSD in Fig. 4a. The fast components have largely been filtered out. We now apply the same filtering to the energetic balanced mode, $\operatorname{BAL}_{(k, 1,324 \mathrm{~m})}$, and also the balanced mode with appropriate phase period $\mathrm{BAL}_{(k, 1,92 \mathrm{~m})}$. For $\mathrm{BAL}_{(k, 1,324 \mathrm{~m})}$, the PSD is illustrated in Fig. 4e, and coherence output in Fig. 4f. The PSD and coherence output of $\operatorname{BAL}_{(k, 1,92 \mathrm{~m})}$ are illustrated in Fig. $4 \mathrm{~g}$ and Fig. 4h, respectively. In both cases, the coherence output is shown to retain only the slow components, as previously demonstrated for the $\mathrm{EIG}_{(k, 0,324 \mathrm{~m})}$ mode. Note, Figs. $4 \mathrm{a}$ and $4 \mathrm{~d}-4 \mathrm{~h}$ all have the same color bar scale.

The coherence output with respect to $\mathrm{EIG}_{(1,0,7 \mathrm{~m})}$ is now calculated for the $\mathrm{EIG}_{\left(1,0, D_{m}\right)}$ modes for all equivalent heights, and illustrated in Fig. 4i. This figure indicates that the slow MJO time scales of the $\operatorname{EIG}_{\left(1,0, D_{m}\right)}$ modes are the most coherent with $\mathrm{EIG}_{(1,0,7 \mathrm{~m})}$. For time scales shorter than 30 days, the coherence output decreases with decreasing time scale (or increasing frequency). The coherence output is organized into two clusters of equivalent heights, the first ranging between $1 \mathrm{~km}$ and $100 \mathrm{~m}$ and the second $100 \mathrm{~m}$ and below. The maxima in these two clusters are associated with the $\mathrm{EIG}_{(1,0,324 \mathrm{~m})}$ and $\mathrm{EIG}_{(1,0,7 \mathrm{~m})}$ modes, respectively.

The associated coherence output for $\mathrm{BAL}_{\left(1,1, D_{m}\right)}$ is illustrated in Fig. 4j. Again, the intraseasonal time scales are the most coherent, with the coherence output decreasing as the frequency increases. The coherence output is again organized into two clusters of equivalent heights, spanning the same ranges observed in the $\mathrm{EIG}_{\left(1,0, D_{m}\right)}$ case. The first cluster ranges from $1 \mathrm{~km}$ to $100 \mathrm{~m}$, is aligned with the secondary peak in energy in Fig. $3 c$, and has a local maximum at $\mathrm{BAL}_{(1,1,324 \mathrm{~m})}$. In Žagar and Franzke (2015) a mode of similar $D_{m}$ was shown to have components correlated with the RMM index of Wheeler and Hendon (2004) with more variance than any of the other NMFs. The second cluster is below $100 \mathrm{~m}$, and has a maxima at $\mathrm{BAL}_{(1,1,92 \mathrm{~m})}$ modes. This cluster could be further split into two considering there are two local maxima. However, we treat them as one group since they have many consistent properties, including similar horizontal structure, time scales within the intraseasonal band, and similar energy levels. For completeness we have also calculated the coherence output for the $\mathrm{WIG}_{\left(1,0, D_{m}\right)}$ modes, and found that they are two orders of magnitude lower than the $\mathrm{EIG}_{\left(1,0, D_{m}\right)}$ and $\mathrm{BAL}_{\left(1,0, D_{m}\right)}$ modes. This suggests that the WIG modes play a negligible role in the evolution of the MJO.

In summary four pertinent mode clusters are identified as interacting with each other, and having properties compatible with the MJO. The cluster centered on the $\operatorname{EIG}_{(1,0,7 \mathrm{~m})}$ mode has a horizontal structure that is equatorially trapped within $15^{\circ} \mathrm{S}-15^{\circ} \mathrm{N}$, and a phase period as determined from the linearized equations of motion to be appropriate for the MJO. This mode has all of the necessary temporal and horizontal properties. In itself, it has low energy, but is coherent with the intraseasonal components of the following more energetic modes. The energetic $\mathrm{EIG}_{(1,0,324 \mathrm{~m})}$ mode cluster has a broader meridional extent, with the HSFs nonzero between approximately $50^{\circ} \mathrm{S}$ and $50^{\circ} \mathrm{N}$. The $\mathrm{BAL}_{(1,1,92 \mathrm{~m})}$ group has an appropriate time scale, but in isolation propagates westward. The $\mathrm{BAL}_{(1,1,324 \mathrm{~m})}$ cluster has a broader meridional extent, and is the most energetic of the aforementioned clusters. However, in isolation this mode propagates too quickly and in the opposite direction as compared to the MJO.

We hypothesize that the nonlinear interaction between each of these mode four groups ensures that a component of the energetic modes propagate eastward with an intraseasonal phase period. A minimal model of the MJO would require the interacting triads formed by at least one mode central to each of the abovementioned clusters. The reduced-order model equations would be a set of ordinary differential equations (ODEs), derived from the projection of these orthogonal modes onto the nonlinear equations of motion, akin to what is done in the reduced-order modeling of canonical fluid flows (Noack et al. 2003; Kitsios 2010). These ODEs would be solved numerically to return the time-varying contribution of each of these modes to the MJO dynamics. To refine the representation of the MJO, the projection could be systematically expanded to include additional equivalent heights radiating out from the aforementioned four modes, and also finer-scale longitudinal waves. This framework incorporates the interactions between 
multiple vertical scales, and also between the tropics and extratropics via the varying meridional extents of the HSFs. Since $\mathrm{EIG}_{(1,0,7 \mathrm{~m})}$ has all of the necessary temporal properties and horizontal structure, we will use this mode to extract the MJO in the following analysis.

\section{Phase averages}

To provide further details on the three-dimensional spatial structure of the MJO, phase averages are constructed using $\mathrm{EIG}_{(1,0,7 \mathrm{~m})}$. Akin to the process undertaken in Wheeler and Hendon (2004) we calculate phase angles from the real and imaginary components of the expansion coefficients $\chi_{k n m}^{q}(t)$, in place of their principal components RMM1 and RMM2. The phases are split into eight discrete octants of indices $j=1-8$, with phase angle ranges defined between $-180^{\circ}+45^{\circ}(j-1)$ and $-135^{\circ}+45^{\circ}(j-1)$. Anomalous daily fields are first calculated about the yearly cycle, and then bandpass filtered to retain the intraseasonal time scales. The anomalous fields associated with the dates in each phase octant are then averaged. Phase average anomalies are then calculated by removing the time-averaged field. While the EIG $_{(1,0,7 \mathrm{~m})}$ mode is used to identify the dates associated with each phase octant, since we average through the daily fields all NMFs are essentially contributing.

The phase average anomalies are illustrated by threedimensional isosurfaces of the velocity potential overlaid with contours of OLR in Fig. 5. To account for the change in magnitude of the velocity potential with level, the fields are normalized by the standard deviations on each level. The dark and light gray structures represent isosurfaces two standard deviations above and below the mean, respectively. From phase 1 to phase 8, this figure demonstrates a consistent eastward propagation of the velocity potential structures, and a dipole type behavior of the OLR field dominant over the Maritime Continent. The phases are also in line with those presented in Wheeler and Hendon (2004). In phase 1 convection from the previous MJO event (positive convection anomaly) is centered over the east of the Maritime Continent. Subsequent phases see convection ramping up in magnitude initially over the east of Africa. There is a strong coupling between the OLR and velocity potential fields in phase 5 with the interface between positive and negative anomalies in both fields occurring at approximately $90^{\circ} \mathrm{E}$. The imprint of the upperlevel velocity potential is also evident outside of the Maritime Continent in the OLR, with anomalies of like sign aligned.

The velocity potential also changes sign in the vertical, which is representative of convection being associated with upper-level divergence (positive velocity potential) and lower-level convergence (negative velocity potential). This is again consistent with the results of Wheeler and Hendon (2004), in which the first two EOFs have their 200-hPa zonal wind component of opposite sign to the 850-hPa zonal wind.

\section{Composite fields}

By construction phase averages, such as those presented in the previous section, contain only spatial structure and no temporal information. A more stringent test is to determine if the spatial and temporal properties of fields composited on the basis of $\mathrm{EIG}_{(1,0,7 \mathrm{~m})}$ are representative of the MJO. The expansion coefficients $\chi_{k n m}^{q}$ are bandpass filtered to retain only the intraseasonal time scales, from which phase angles are then calculated. The phase angle time series is split into a series of events, demarcated by a discontinuous change in phase angle. A discontinuity is defined as when the phase angle changes by more than $90^{\circ}$ in one day. To select the strong and persistent events, we retain only those that propagate for at least $270^{\circ}$ and have an average magnitude in the upper quartile. Over the 1958-2016 period, 96 events are identified, with on average $1.6 \mathrm{MJO}$ events identified per year. The alignment of each event is defined using only the phase information, and hence not explicitly dependent upon the magnitude. Day 0 of each event is defined as the day in which a least squares fit to the phase angle passes through $0^{\circ}$. This is by definition associated with the transition from phase 4 to phase 5 as presented in section 6.

The magnitude of the selected events is illustrated as the faint gray lines in Fig. 6a. The ensemble mean and standard deviation is calculated for each day offset from day 0 . The solid bold line in this figure is the ensembleaveraged magnitude, with the dashed lines showing the ensemble average plus and minus one ensemble standard deviation. There is a local maximum in magnitude in the vicinity of day 0 , which indicates that these events are stronger than the background variability of this mode. The ensemble-averaged phase angles are illustrated in Fig. 6b, and indicate a persistent eastward propagation.

To confirm the spatial structure, ensemble-averaged composites of velocity potential, OLR, and precipitation are calculated for the days leading up to and following day 0 . First, anomalous daily fields are calculated to have the yearly cycle removed and also ENSO time scales filtered out. The fields of dates associated with day 0 are averaged together to produce an ensembleaveraged day 0 . Likewise, the same process is applied to produce ensemble-averaged composites for the nonzero day shifts. The ensemble-averaged velocity potential 
(a) phase 1

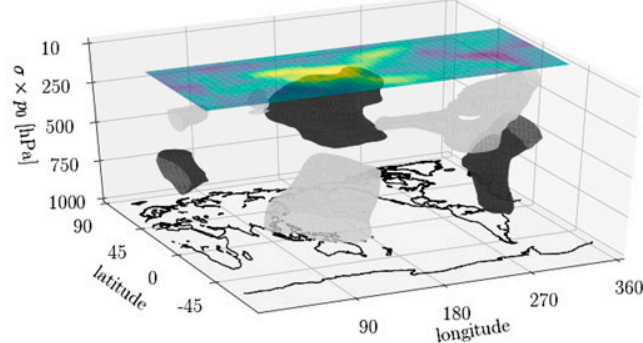

(c) phase 3

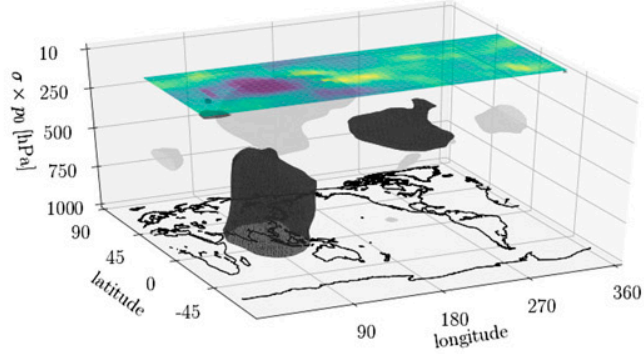

(e) phase 5

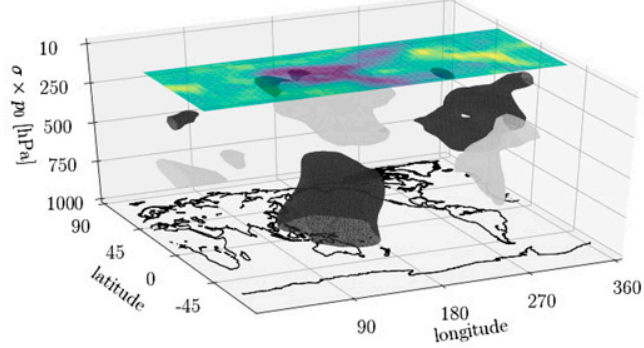

(g) phase 7

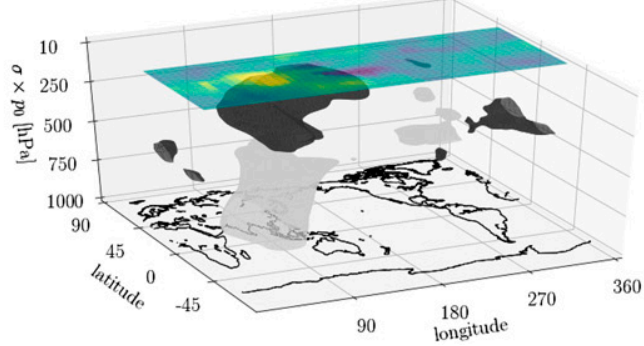

(b) phase 2

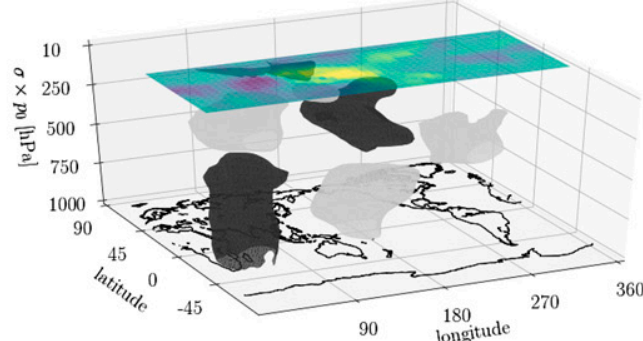

(d) phase 4

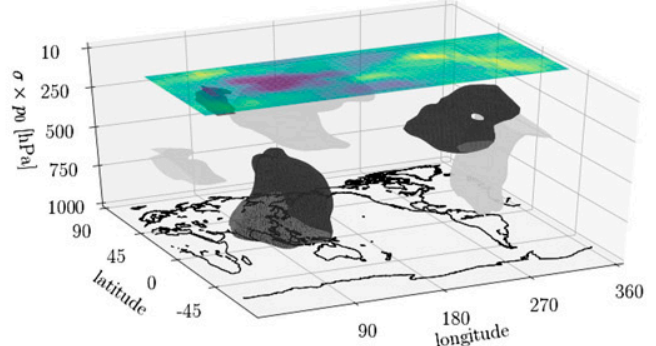

(f) phase 6

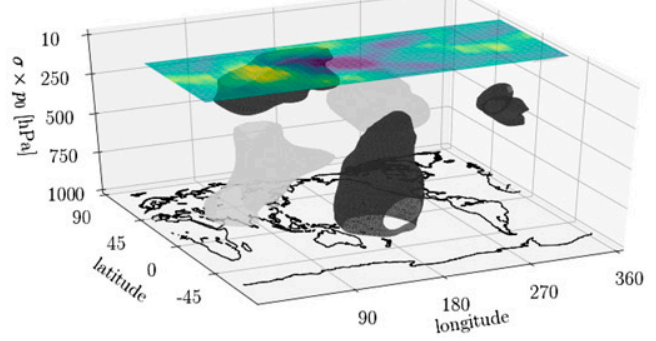

(h) phase 8

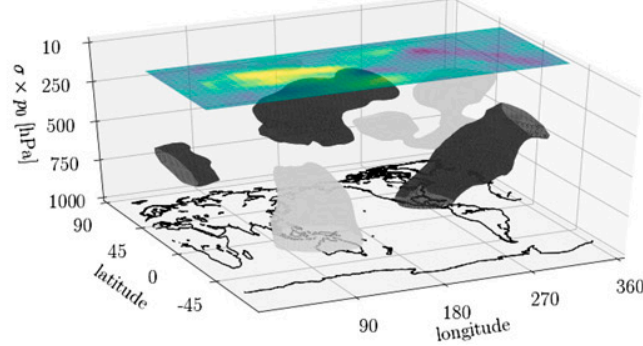

FIG. 5. Anomalies of phase-averaged OLR and velocity potential fields, calculated using the EIG $\left(k, n, D_{m}\right)=(1,0,7 \mathrm{~m})$ mode of phases angles: (a) phase 1 from $-180^{\circ}$ to $-135^{\circ}$, (b) phase 2 from $-135^{\circ}$ to $-90^{\circ}$, (c) phase 3 from $-90^{\circ}$ to $-45^{\circ}$, (d) phase 4 from $-45^{\circ}$ to $0^{\circ}$, (e) phase 5 from $0^{\circ}$ to $45^{\circ}$, (f) phase 6 from $45^{\circ}$ to $90^{\circ}$, (g) phase 7 from $90^{\circ}$ to $135^{\circ}$, and (h) phase 8 from $135^{\circ}$ to $180^{\circ}$. OLR contours range from -4 (blue) to $4 \mathrm{~W} \mathrm{~m}^{-2}$ (yellow). Velocity potential isosurfaces are shaded for two standard deviations below the mean (light gray) and two standard deviations above the mean (dark gray). The vertical axis, $\sigma \times p_{0}$, is an indicative pressure where $p_{0}=1013 \mathrm{hPa}$.

at a pressure level of $200 \mathrm{hPa}$ is illustrated in Fig. 7 for a selection of days from 20 days before to 20 days after. There is a clear propagation of the structures over this 41-day period. The ensemble-averaged OLR is illustrated in Fig. 8 over the same relative day range. The OLR fields demonstrate a dipole type oscillation over the Maritime Continent. Composites of precipitation are illustrated in Fig. 9, which demonstrates that this field has structures very similar to those observed in the OLR fields, but of opposite sign. The observed strong anticorrelation 

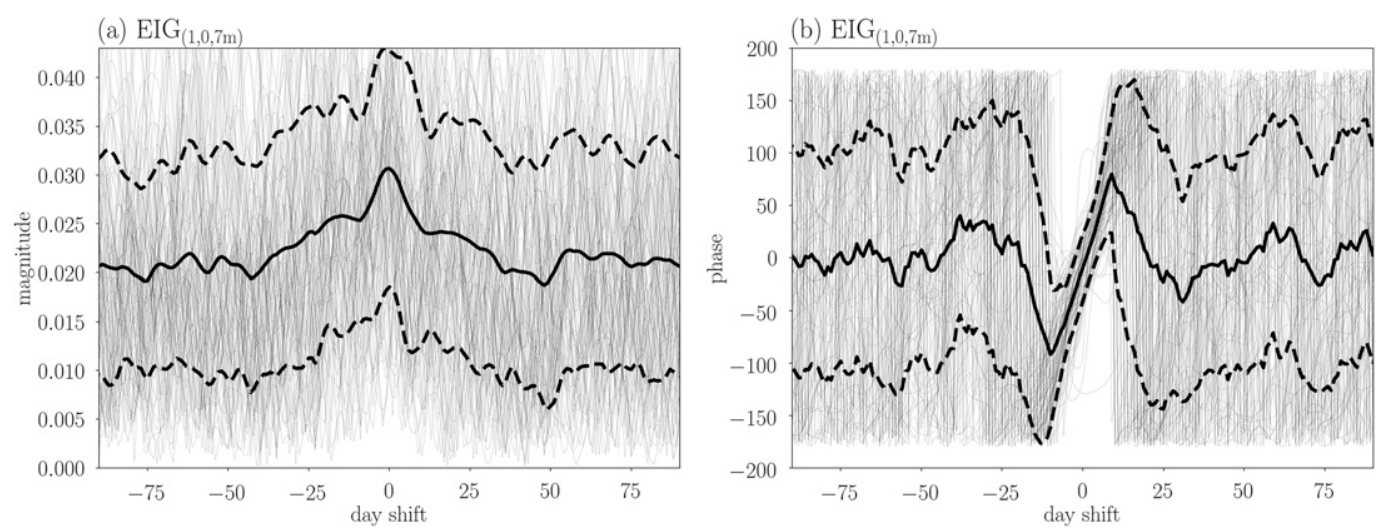

FIG. 6. Composite-averaged normal mode function complex expansion coefficients $\chi_{k n m}^{q}$ : (a) magnitude of the EIG mode triplet $\left(k, n, D_{m}\right)=(1,0,7 \mathrm{~m})$ relative to day 0 , illustrating each individual event (faint lines), the ensemble mean (thick solid line) and plus and minus one ensemble standard deviation (thick dashed lines); and (b) as in (a), but for the phase angle.

between these two fields is consistent with the view that anomalously low (high) OLR leads to high (low) temperatures and increased (decreased) evaporation and water vapor concentration, which finally produces anomalously high (low) rainfall. By design the structure at day 0 , of approximate phase angle $0^{\circ}$, is similar to that of phase 5 illustrated in Fig. 5e. Consistent with Fig. 6a, the magnitude of the velocity potential, OLR, and precipitation fields all decrease the further in time they are away from day 0 .

\section{Characterization of individual MJO events}

We next contrast the NMF MJO characterization with the RMM index of Wheeler and Hendon (2004). The evolution of the NMF energy $E_{k n m}^{q}$ is illustrated for the modes central to the four clusters identified in section 5 . To recap, these clusters in order of decreasing energy content are the energetic Rossby waves, the energetic EIG waves, the Rossby waves of intraseasonal phase period, and the EIG waves of appropriate phase period. The modes central to each of the above groups are $\operatorname{BAL}_{(1,1,324 \mathrm{~m})}, \mathrm{EIG}_{(1,0,324 \mathrm{~m})}, \mathrm{BAL}_{(1,1,92 \mathrm{~m})}$, and $\mathrm{EIG}_{(1,0,7 \mathrm{~m})}$, respectively.

Akin to what is done in Wheeler and Hendon (2004), 91-day centered moving averages are calculated. The square root of these moving averages are illustrated in Fig. 10a for the period from 2000 to 2016 . The $\mathrm{BAL}_{(1,1,324 \mathrm{~m})}$ mode is indicated by the red dotted line. These coefficients do not appear to follow the RMM index particularly well. It has a correlation coefficient with RMM of 0.42 over the entire period from 1958 to 2016 . However, the agreement improves significantly when filtered to retain only the temporal scales that have a coherence with $\mathrm{EIG}_{(1,0,7 \mathrm{~m})}$ greater than 0.5 . The filtered time series is illustrated by the solid red line, and has a correlation coefficient with RMM of 0.78 . In effect, filtering on the basis of $\mathrm{EIG}_{(1,0,7 \mathrm{~m})}$ removes all negative-frequency contributions (i.e., westward-propagating waves), low-frequency time scales, and high-frequency weather. The following NMFs, also illustrated in Fig. 10a, are discussed in order of decreasing energy content and correlation. The $\mathrm{EIG}_{(1,0,324 \mathrm{~m})}$ mode is filtered in the aforementioned manner and has a correlation with RMM of 0.82 . Likewise, the filtered $\mathrm{BAL}_{(1,1,92 \mathrm{~m})}$ mode has a correlation of 0.62 . Finally the unfiltered $\mathrm{EIG}_{(1,0,7 \mathrm{~m})}$ mode has a correlation with RMM of 0.32 . This mode has the least energy contribution, but is required to isolate the MJO component in the more energetic modes.

From the perspective of the vertical structure, the high energy modes, $\mathrm{BAL}_{(1,1,324 \mathrm{~m})}$ and $\mathrm{EIG}_{(1,0,324 \mathrm{~m})}$, have an MJO-appropriate baroclinic structure for altitudes lower than $100 \mathrm{hPa}$, but an additional six zero crossings within the stratosphere. This is illustrated by the associated VSF in Fig. 1c. The VSF associated with the $\mathrm{EIG}_{(1,0,7 \mathrm{~m})}$ mode, however, is essentially zero above $100 \mathrm{hPa}$, also illustrated in Fig. 1c. Filtering on the basis of $\mathrm{EIG}_{(1,0,7 \mathrm{~m})}$ removes the higher-altitude, non-MJO-related dynamics.

We have identified four MJO events defined as local maxima of the RMM index, which in both order of increasing magnitude and chronological order are in November 2002, January 2006, February 2008, and March 2012. Note these dates also correspond to local maxima in our $\mathrm{BAL}_{(1,1,324 \mathrm{~m})}$ mode. The event in March 2012 is the largest-magnitude event within the 2000-16 period. Each of these events are highlighted by the yellow box in Fig. 10a. During the November 2002 event, Fig. 10b illustrates that the phase angle for the coherence-filtered $\operatorname{BAL}_{(1,1,324 \mathrm{~m})}$ mode and the RMM index and exhibit very 
(a) -20 days $;(\min , \max )=(-1.6,2.5) \times 10^{6} \mathrm{~m}^{2} \mathrm{~s}^{-1}$

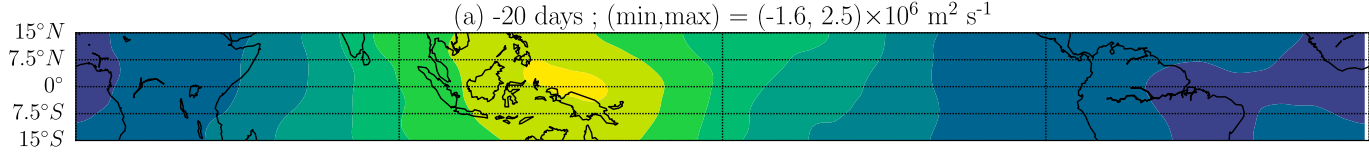

(b) -15 days $;(\min , \max )=(-2.6,4.2) \times 10^{6} \mathrm{~m}^{2} \mathrm{~s}^{-1}$

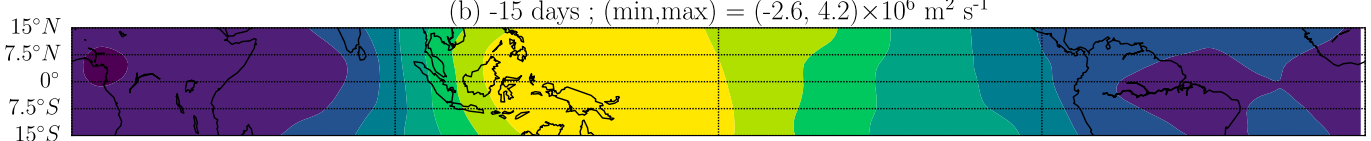

(c) -10 days $;(\min , \max )=(-4.8,3.8) \times 10^{6} \mathrm{~m}^{2} \mathrm{~s}^{-1}$

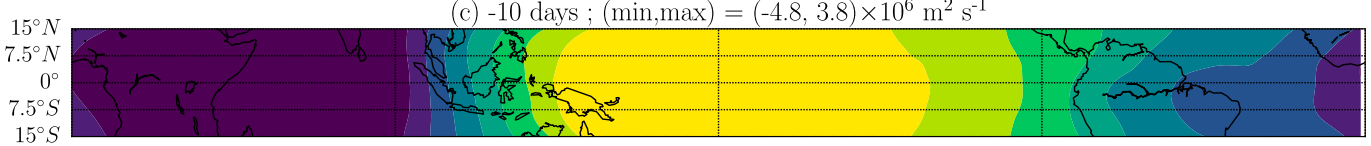

(d) -5 days $;(\min , \max )=(-5.9,4.2) \times 10^{6} \mathrm{~m}^{2} \mathrm{~s}^{-1}$

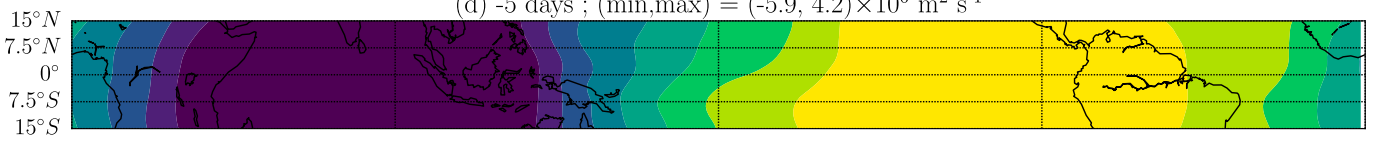

(e) 0 days $;(\min , \max )=(-6.1,4.7) \times 10^{6} \mathrm{~m}^{2} \mathrm{~s}^{-1}$

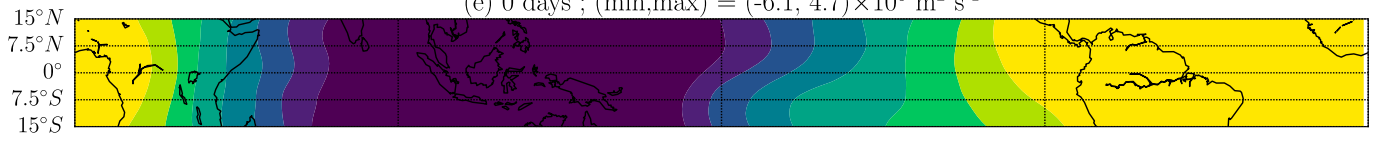

(f) 5 days; $(\min , \max )=(-5.2,4.8) \times 10^{6} \mathrm{~m}^{2} \mathrm{~s}^{-1}$

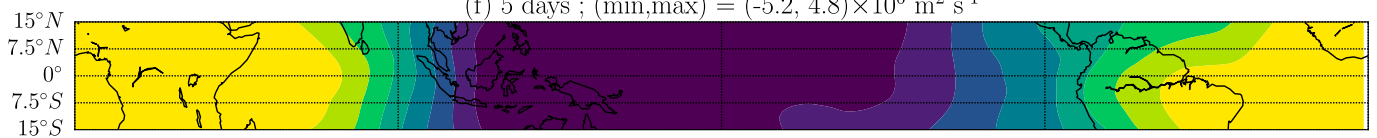

(g) 10 days; $(\min , \max )=(-3.0,5.4) \times 10^{6} \mathrm{~m}^{2} \mathrm{~s}^{-1}$
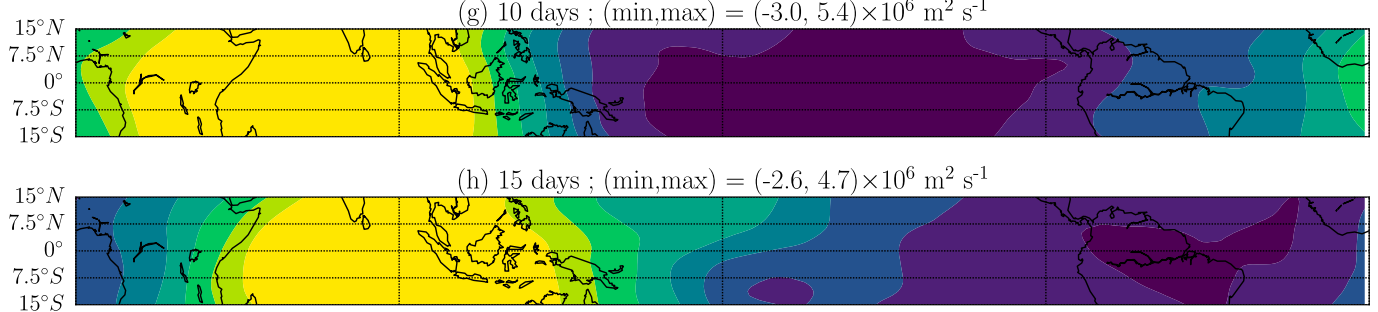

$15^{\circ} \mathrm{N}$ (i) 20 days ; $(\min , \max )=(-2.3,3.0) \times 10^{6} \mathrm{~m}^{2} \mathrm{~s}^{-1}$

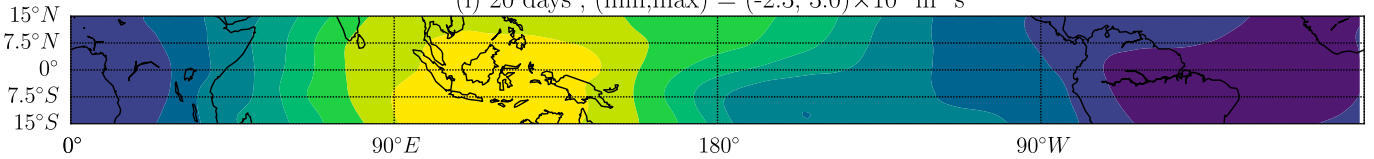

FIG. 7. Composite-averaged velocity potential field at $200 \mathrm{hPa}$ based upon the $\mathrm{EIG}\left(k, n, D_{m}\right)=(1,0,7 \mathrm{~m})$ mode for days relative to day 0: (a) 20 days before, (b) 15 days before, (c) 10 days before, (d) 5 days before, (e) day 0 , (f) 5 days after, (g) 10 days after, (h) 15 days after, and (i) 20 days after. Contour values in all plots range from $-2.5 \times 10^{6}$ (blue) to $2.5 \times 10^{6} \mathrm{~m}^{2} \mathrm{~s}^{-1}$ (yellow). Minimum ( $\left.\mathrm{min}\right)$ and maximum values (max) over the illustrated domain for each plot are listed in their respective titles.

similar phase propagation. This is also true for the phase propagation of the January 2006, February 2008, and March 2012 events illustrated in Figs. 10c, 10d, and 10e, respectively. For the March 2012 event, the phase portrait of the RMM index illustrated in Fig. 10f demonstrates a counterclockwise phase progression of relatively consistent magnitude. The associated phase portrait of the coherence-filtered $\mathrm{BAL}_{(1,1,324 \mathrm{~m})}$ mode is illustrated in Fig. 10g, and demonstrates a similar counterclockwise evolution. In summary both the magnitude and the phase of the coherence-filtered energetic $\mathrm{BAL}_{(1,1,324 \mathrm{~m})}$ mode are very similar to those of the widely accepted RMM index of Wheeler and Hendon (2004).

\section{Concluding remarks}

The MJO is described here as a combination of interacting balanced (BAL) modes (i.e., Rossby waves) and eastward-propagating inertial gravity (EIG) waves of varying vertical scale and meridional extent. The MJO 


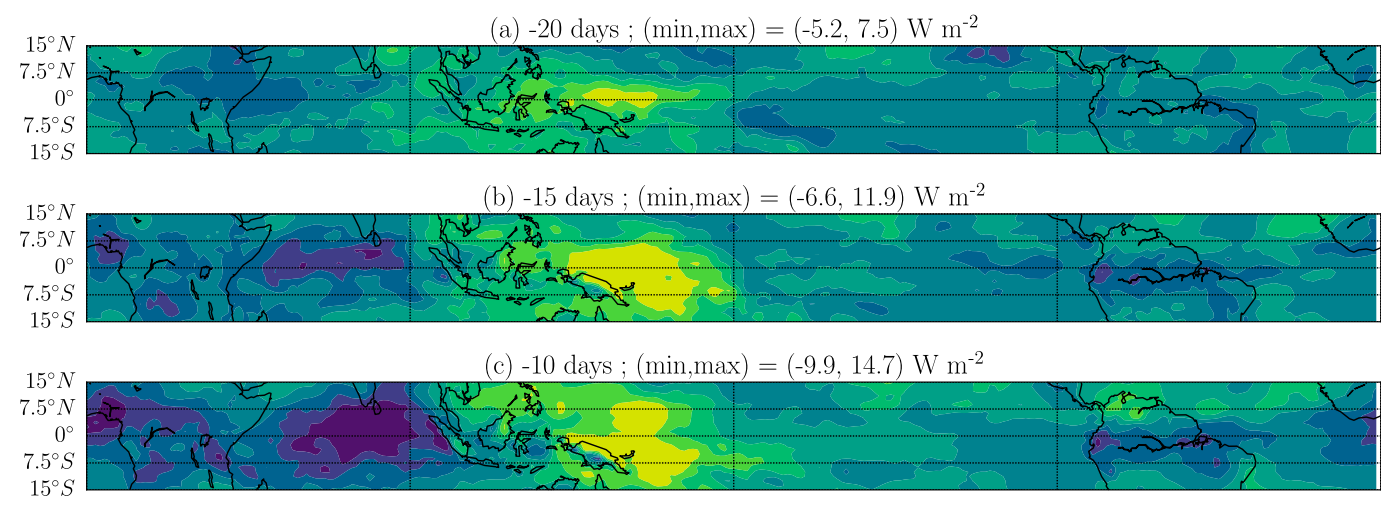

(d) -5 days $;(\min , \max )=(-13.2,10.0) \mathrm{W} \mathrm{m}^{-2}$

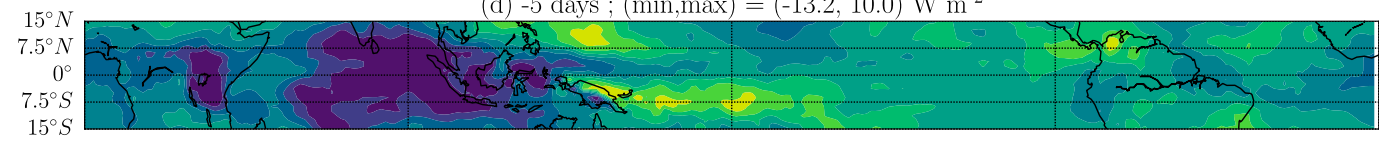

(e) 0 days $;(\min , \max )=(-13.2,9.3) \mathrm{W} \mathrm{m}^{-2}$
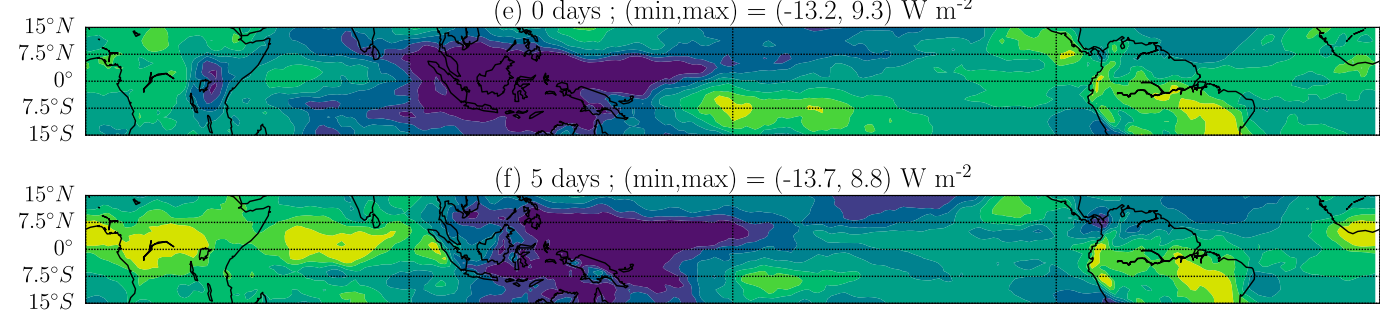

(g) 10 days $;(\min , \max )=(-11.8,12.0) \mathrm{W} \mathrm{m}^{-2}$

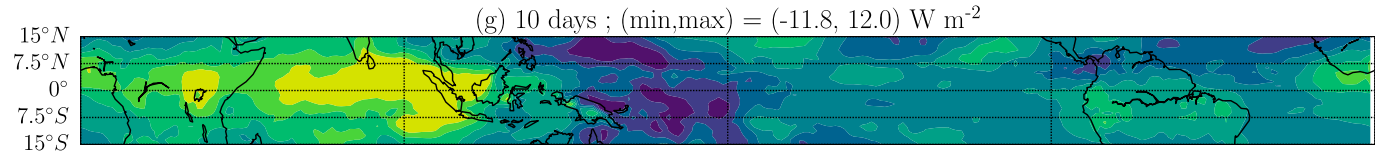

(h) 15 days ; $(\min , \max )=(-11.5,9.3) \mathrm{W} \mathrm{m}^{-2}$

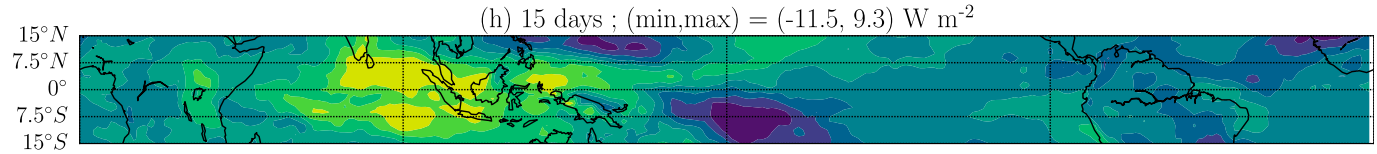

(i) 20 days ; $(\min , \max )=(-9.3,8.4) \mathrm{W} \mathrm{m}^{-2}$

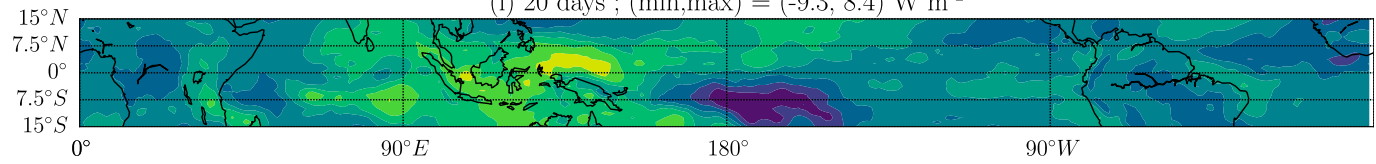

FIG. 8. Composite-averaged outgoing longwave radiation field based upon the EIG $\left(k, n, D_{m}\right)=(1,0,7 \mathrm{~m})$ mode for days relative to day 0: (a) 20 days before, (b) 15 days before, (c) 10 days before, (d) 5 days before, (e) day 0 , (f) 5 days after, (g) 10 days after, (h) 15 days after, and (i) 20 days after. Contour values in all plots range from -8 (blue) to $8 \mathrm{~W} \mathrm{~m}^{-2}$ (yellow). Min and max values over the illustrated domain for each plot are listed in their respective titles.

was isolated from JRA-55 via a normal mode function (NMF) scale and mode class decomposition. The only NMFs that have the appropriate MJO-like horizontal structure are the Rossby wave BAL modes of zonal wavenumber $k=1$ and meridional index $n=1$, and the EIG modes of $(k, n)=(1,0)$. EIG modes with $n=0$ are by definition Kelvin waves. The potential for the nonlinear interactions between these modes was quantified by the coherence output, which identified four distinct interacting clusters of modes spanning various vertical scales. These groups are listed below in order of increasing energy content. The first group consists of Kelvin waves, with a maximum coherence output occurring at an equivalent height of $7 \mathrm{~m}$. The modes in this cluster propagate eastward, have eigenvaluedetermined time scales within the intraseasonal band, are equatorially trapped between $15^{\circ} \mathrm{S}$ and $15^{\circ} \mathrm{N}$, but have minimal energy as compared to the following mode groups. This is perhaps dynamically the most important cluster since it is the only one to have all of the 


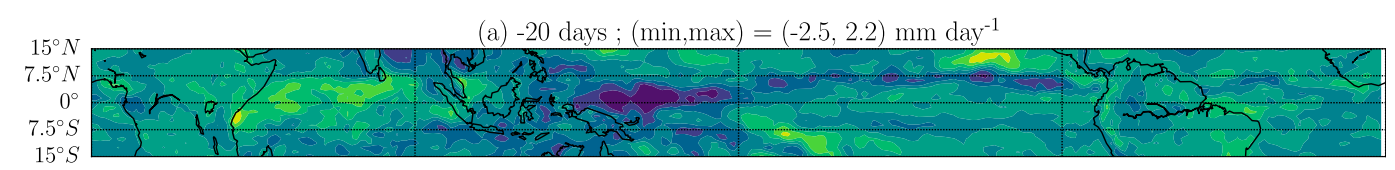

(b) -15 days $;(\min , \max )=(-3.6,3.8) \mathrm{mm}$ day $^{-1}$

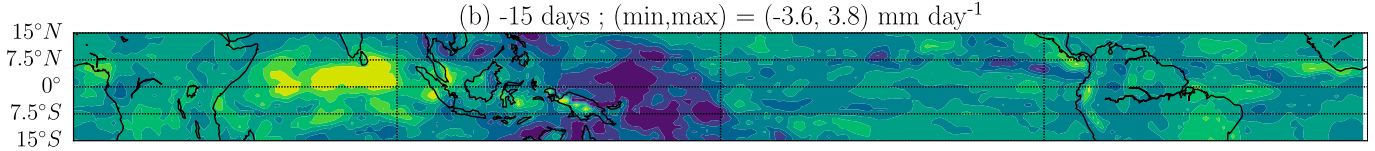

(c) -10 days $;(\min , \max )=(-5.7,6.3) \mathrm{mm}_{\text {day }}{ }^{-1}$

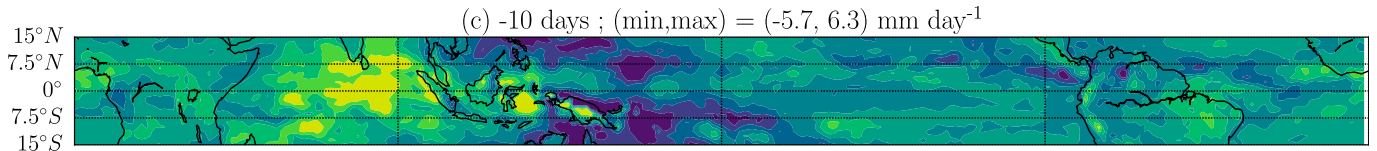

(d) -5 days $;(\min , \max )=(-3.9,5.6) \mathrm{mm}_{\text {day }}{ }^{-1}$

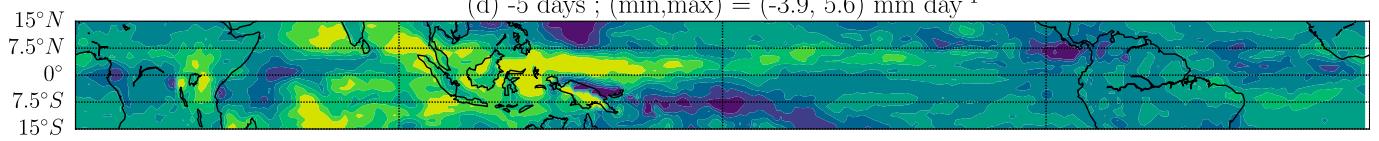

(e) 0 days $;(\min , \max )=(-3.0,4.2) \mathrm{mm}_{\text {day }}{ }^{-1}$

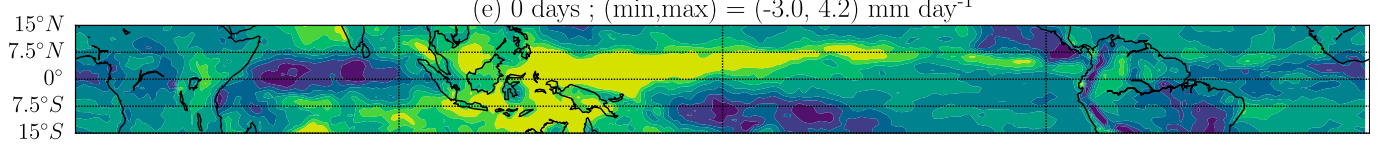

(f) 5 days $;(\min , \max )=(-3.6,3.7) \mathrm{mm}$ day $^{-1}$

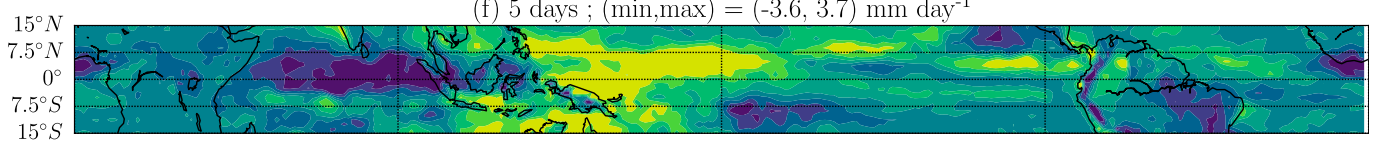

(g) 10 days $;(\min , \max )=(-4.6,3.3) \mathrm{mm} \mathrm{day}^{-1}$

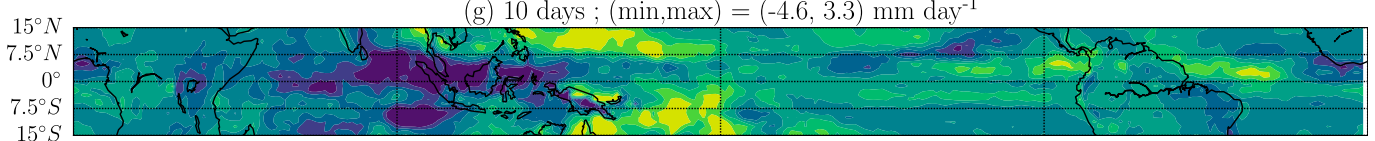

(h) 15 days $;(\min , \max )=(-3.3,3.7) \mathrm{mm}_{\text {day }}{ }^{-1}$

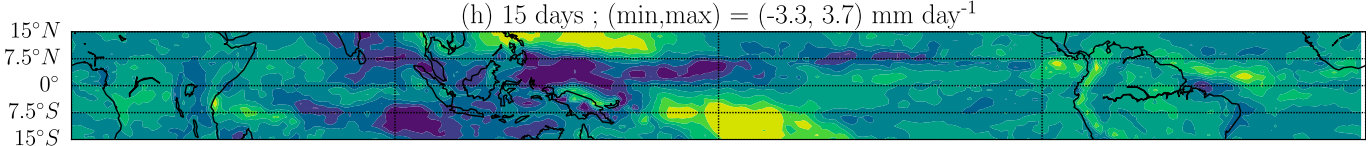

(i) 20 days $;(\min , \max )=(-2.8,3.4) \mathrm{mm} \mathrm{day}^{-1}$

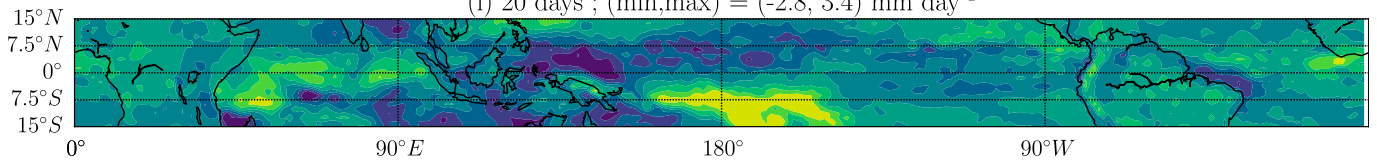

FIG. 9. Composite-averaged precipitation field based upon the $\mathrm{EIG}\left(k, n, D_{m}\right)=(1,0,7 \mathrm{~m})$ mode for days relative to day 0: (a) 20 days before, (b) 15 days before, (c) 10 days before, (d) 5 days before, (e) day 0, (f) 5 days after, (g) 10 days after, (h) 15 days after, and (i) 20 days after. Contour values in all plots range from -2 (blue) to $2 \mathrm{~mm} \mathrm{day}^{-1}$ (yellow). Min and max values over the illustrated domain for each plot are listed in their respective titles.

MJO-appropriate horizontal and temporal properties. The second group consists of Rossby waves with the coherence output maxima located at an equivalent height equal of $92 \mathrm{~m}$. They also have the appropriate time scales and reasonable energy, but in isolation would propagate westward (as governed by their eigensolution). The third group contains the most energetic Kelvin waves with the equivalent height of $324 \mathrm{~m}$ having the highest coherence. These modes also encompass the extratropics with a broader meridional extent from $50^{\circ} \mathrm{S}$ to $50^{\circ} \mathrm{N}$, but have time scales that are too short. The final group is the most energetic and contains Rossby waves with the same equivalent height, and similar meridional extents as the previous group. Their time scales are again too short and also propagate westward.

A cross-spectral analysis indicated that the above mode groups are coherent and presumably interact with one another. The only way that these orthogonal NMFs can interact is through nonlinear triads. We, therefore, hypothesize that the nonlinear interaction between the above mode groups conspire such that on average the energetic modes propagate eastward with an intraseasonal time scale, as observed. In this 

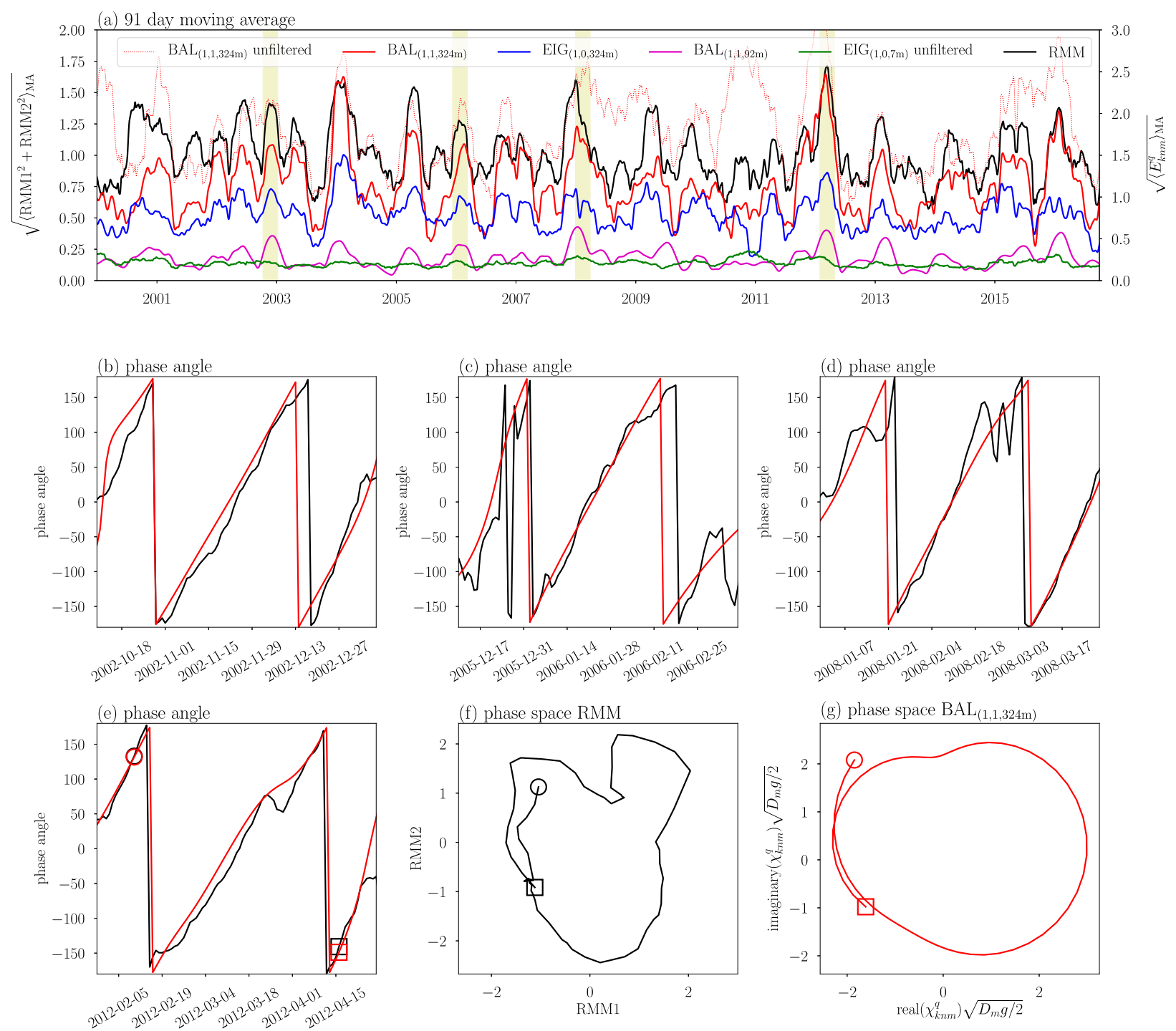

FIG. 10. MJO time series: (a) square root of the 91-day centered moving averages $\left(\sqrt{\langle\cdot\rangle_{\mathrm{MA}}}\right)$ of the RMM index $\left(\mathrm{RMM} 1^{2}+\mathrm{RMM} 2^{2}\right)$, the NMF energy $E_{k n m}^{q}$ of unfiltered modes $\mathrm{BAL}_{(1,1,324 \mathrm{~m})}$ and $\mathrm{EIG}_{(1,0,7 \mathrm{~m})}$, and coherence-filtered modes $\mathrm{BAL}_{(1,1,324 \mathrm{~m})}$, $\mathrm{EIG}_{(1,0,324 \mathrm{~m})}$, and BAL $_{(1,1,92 \mathrm{~m})}$, with the yellow boxes highlighting the time periods illustrated in the remaining plots; (b) comparison of the phase angles of RMM and the coherence-filtered BAL $\mathrm{BL}_{(1,1,324 \mathrm{~m})}$ mode for an MJO event in November 2002; (c) as in (b), for January 2006; (d) as in (b), for February 2008; (e) as in (b), for March 2012; (f) RMM in phase space for the March 2012 event; and (g) coherence-filtered BAL $(1,1,324 \mathrm{~m})$ mode in phase space for the March 2012 event. In (e)-(g), the circle and square markers indicate the start and end of the period of interest, respectively. The legend in (a) is applicable to all plots.

representation of the $\mathrm{MJO}$ a minimal set of interacting triads is formed by the Kelvin waves $\operatorname{EIG}_{\left(k, n, D_{m}\right)}=$ $\mathrm{EIG}_{(1,0,7 \mathrm{~m})}$ and $\mathrm{EIG}_{(1,0,324 \mathrm{~m})}$, and the Rossby waves $\mathrm{BAL}_{(1,1,92 \mathrm{~m})}$ and $\mathrm{BAL}_{(1,1,324 \mathrm{~m})}$. A reduced-order model of the MJO could be developed in the future by projecting these modes onto the nonlinear equations of motion, and solving numerically for the time-varying coefficients of these modes. The MJO dynamics could be made more complete by expanding the range of vertical modes included in the projection. Finer-scale longitudinal waves could also be included. This framework captures the interactions within a hierarchy of vertical scales. Interactions between the tropics and extratropics are also included, since the horizontal structure functions extend farther into the extratropics as their equivalent height increases.

On the basis of the $\mathrm{EIG}_{(1,0,7 \mathrm{~m})}$ Kelvin wave, phase averages and composites of large and persistent MJO events were calculated. Both analyses demonstrate the eastward propagation of the velocity potential and the dipole-like oscillation of outgoing longwave radiation and precipitation over the Maritime Continent. 
The composite analysis also indicates that this reference Kelvin wave isolates the appropriate intraseasonal time scales. The time-scale components of the more energetic modes coherent with $\mathrm{EIG}_{(1,0,7 \mathrm{~m})}$ are also shown to be highly correlated with the accepted MJO index of Wheeler and Hendon (2004).

These findings may in some way explain the influence of vertical resolution on the representation of the MJO in general circulation models (GCMs). Crueger et al. (2013) undertook a meta-analysis of 42 experiments of varying configuration and resolution, including both atmospheric and coupled GCMs. One of their key findings was that eastward propagation was enhanced when vertical and horizontal resolution were increased together, while changing only vertical resolution had a lesser influence. However, as stated in Liess and Bengtsson (2004), the relative lesser importance of the vertical grid could be due to having an inappropriate ratio of vertical to horizontal resolution. Regardless of the quantum of the influence of the vertical grid, we speculate that associated improvements in the representation of the MJO is related to the importance of the $\mathrm{EIG}_{(1,0,7 \mathrm{~m})}$ mode within this NMF framework. This mode has a small equivalent height of $7 \mathrm{~m}$ and its vertical structure has oscillations distributed throughout the troposphere-see Fig. 1c. If this mode is not explicitly resolved on the vertical grid, then the mechanism for changing the direction and slowing down the energetic Rossby waves would be broken, and hence the MJO would be poorly represented.

Acknowledgments. The authors were supported by the Australian Commonwealth Scientific and Industrial Research Organisation Decadal Forecasting Project (research.csiro.au/dfp). The authors would also like to acknowledge NCI for providing the computational resources.

\section{REFERENCES}

Castanheira, J., and C. Marques, 2015: Convectively coupled equatorial-wave diagnosis using three-dimensional normal modes. Quart. J. Roy. Meteor. Soc., 141, 2776-2792, https:// doi.org/10.1002/qj.2563.

Chang, C., and H. Lim, 1988: Kelvin wave-CISK: A possible mechanism for the 30-50 day oscillations. J. Atmos. Sci., 45, 1709-1720, https://doi.org/10.1175/1520-0469(1988)045<1709: $\mathrm{KWCAPM}>2.0 . \mathrm{CO} ; 2$.

Chen, S., R. Houze Jr., and B. Mapes, 1996: Multiscale variability of deep convection in relation to large-scale circulation in TOGA COARE. J. Atmos. Sci., 53, 1380-1409, https://doi.org/ 10.1175/1520-0469(1996)053<1380:MVODCI>2.0.CO;2.

Chen, S., A. Majda, and S. Stechmann, 2016: Tropicalextratropical interactions with the MJO skeleton and climatological mean flow. J. Atmos. Sci., 73, 4101-4116, https:// doi.org/10.1175/JAS-D-16-0041.1.
Crueger, T., B. Stevens, and R. Brokopf, 2013: The Madden-Julian oscillation in ECHAM6 and the introduction of an objective MJO metric. J. Climate, 26, 3241-3257, https://doi.org/10.1175/ JCLI-D-12-00413.1.

DeMott, C., N. Klingaman, and S. Woolnough, 2015: Atmosphereocean coupled processes in the Madden-Julian oscillation. Rev. Geophys., 53, 1099-1154, https://doi.org/10.1002/2014RG000478.

Emanuel, K., 1987: An air-sea interaction model of intraseasonal oscillations in the tropics. J. Atmos. Sci., 44, 2324-2340, https://doi.org/10.1175/1520-0469(1987)044<2324:AASIMO> 2.0.CO;2.

Frederiksen, J. S., 2002: Genesis of intraseasonal oscillations and equatorial waves. J. Atmos. Sci., 59, 2761-2781, https://doi.org/ 10.1175/1520-0469(2002)059<2761:GOIOAE > 2.0.CO;2.

, and C. Frederiksen, 1993: Monsoon disturbances, intraseasonal oscillations, teleconnection patterns, blocking, and storm tracks of the global atmosphere during January 1979: Linear theory. J. Atmos. Sci., 50, 1349-1372, https://doi.org/ 10.1175/1520-0469(1993)050<1349:MDIOTP > 2.0.CO;2.

, and H. Lin, 2013: Tropical-extratropical interactions of intraseasonal oscillations. J. Atmos. Sci., 70, 3180-3197, https:// doi.org/10.1175/JAS-D-12-0302.1.

Fuchs, Z., and D. J. Raymond, 2005: Large-scale modes in a rotating atmosphere with radiative-convective instability and WISHE. J. Atmos. Sci., 62, 4084-4094, https://doi.org/10.1175/JAS3582.1.

Hendon, H., and M. Salby, 1994: The life cycle of the MaddenJulian oscillation. J. Atmos. Sci., 51, 2225-2237, https://doi.org/ 10.1175/1520-0469(1994)051<2225:TLCOTM>2.0.CO;2.

Kasahara, A., and K. Puri, 1981: Spectral representation of threedimensional global data by expansion in normal mode functions. Mon. Wea. Rev., 109, 37-51, https://doi.org/10.1175/15200493(1981)109<0037:SROTDG > 2.0.CO;2.

Kitsios, V., 2010: Recovery of fluid mechanical modes in unsteady separated flows. Ph.D. thesis, University of Melbourne and Université de Poitiers, 259 pp., http://repository.unimelb.edu.au/ $10187 / 9088$

Kobayashi, A., and Coauthors, 2015: The JRA-55 reanalysis: General specifications and basic characteristics. J. Meteor. Soc. Japan, 93, 5-48, https://doi.org/10.2151/jmsj.2015-001.

Lau, K., and L. Peng, 1987: Origin of low-frequency (intraseasonal) oscillations in the tropical atmosphere. Part I: Basic theory. J. Atmos. Sci., 44, 950-972, https://doi.org/10.1175/15200469(1987)044<0950:OOLFOI > 2.0.CO;2.

Liess, S., and L. Bengtsson, 2004: The intraseasonal oscillation in ECHAM4 part II: Sensitivity studies. Climate Dyn., 22, 671-688, https://doi.org/10.1007/s00382-004-0407-z.

Lindzen, R., 1974: Wave-CISK in the tropics. J. Atmos. Sci., 31, 156-179, https://doi.org/10.1175/1520-0469(1974)031<0156: WCITT $>2.0 . \mathrm{CO} ; 2$.

Madden, R. A., and P. R. Julian, 1971: Detection of a 40-50 day oscillation in the zonal wind in the tropical pacific. J. Atmos. Sci., 28, 702-708, https://doi.org/10.1175/1520-0469(1971) 028<0702:DOADOI $>2.0$.CO;2.

- , and 1972: Description of global-scale circulation cells in the tropics with a 40-50 day period. J. Atmos. Sci., 29, 1109-1123, https://doi.org/10.1175/1520-0469(1972)029<1109: DOGSCC $>2.0 . \mathrm{CO} ; 2$.

Majda, A., and S. Stechmann, 2009: The skeleton of tropical intraseasonal oscillations. Proc. Natl. Acad. Sci. USA, 106, 8417-8422, https://doi.org/10.1073/pnas.0903367106.

Nakazawa, T., 1988: Tropical super clusters within intraseasonal variations over the western Pacific. J. Meteor. Soc. Japan, 66, 823-836, https://doi.org/10.2151/jmsj1965.66.6_823. 
Neelin, J., and I. Held, 1987: Modeling tropical convergence based on the moist static energy budget. Mon. Wea. Rev., 115, 3-12, https://doi.org/10.1175/1520-0493(1987)115<0003:MTCBOT> 2.0.CO;2.

__ , and J.-Y. Yu, 1994: Modes of tropical variability under convective adjustment and the Madden-Julian oscillation. Part I: Analytical theory. J. Atmos. Sci., 51, 1876-1984, https://doi.org/10.1175/1520-0469(1994)051<1876:MOTVUC> 2.0.CO;2.

Noack, B., K. Afanasiev, M. Morzynski, G. Tadmor, and F. Thiele, 2003: A hierarchy of low-dimensional models for the transient and post-transient cylinder wake. J. Fluid Mech., 497, 335-363, https://doi.org/10.1017/S0022112003006694.

Raymond, D. J., and Ž. Fuchs, 2009: Moisture modes and the Madden-Julian oscillation. J. Atmos. Sci., 22, 3031-3046, https://doi.org/10.1175/2008JCLI2739.1.

Rui, H., and B. Wang, 1990: Development characteristics and dynamic structure of tropical intraseasonal convection anomalies. J. Atmos. Sci., 47, 357-379, https://doi.org/10.1175/15200469(1990)047<0357:DCADSO > 2.0.CO;2.

Sobel, A., and E. Maloney, 2012: Moisture modes and the eastward propagation of the MJO. J. Atmos. Sci., 69, 1691-1705, https:// doi.org/10.1175/JAS-D-11-0118.1.

Wang, B., and H. Rui, 1990: Dynamics of the coupled moist Kelvin-Rossby wave on an equatorial $\beta$-plane. J. Atmos. Sci., 47, 397-413, https://doi.org/10.1175/1520-0469(1990)047<0397: DOTCMK $>2.0 . \mathrm{CO} ; 2$.

- and T. Li, 1994: Convective interaction with boundary-layer dynamics in the development of the tropical intraseasonal system. J. Atmos. Sci., 51, 1386-1400, https://doi.org/10.1175/ 1520-0469(1994)051<1386:CIWBLD>2.0.CO;2.

Wheeler, M., and G. Kiladis, 1999: Convectively coupled equatorial waves: Analysis of clouds and temperature in the wavenumberfrequency domain. J. Atmos. Sci., 56, 374-399, https://oi.org/ 10.1175/1520-0469(1999)056<0374:CCEWAO > 2.0.CO;2.

—_, and H. Hendon, 2004: An all-season real-time multivariate MJO index: Development of an index for monitoring and prediction. Mon. Wea. Rev., 132, 1917-1932, https://doi.org/ 10.1175/1520-0493(2004)132<1917:AARMMI > 2.0.CO;2.

Yano, J.-I., and J. Tribbia, 2017: Tropical atmospheric MaddenJulian oscillation: A strongly nonlinear free solitary Rossby wave. J. Atmos. Sci., 74, 3473-3489, https://doi.org/10.1175/ JAS-D-16-0319.1.

Žagar, N., and C. Franzke, 2015: Systematic decomposition of the Madden-Julian oscillation into balanced and inertio-gravity components. Geophys. Res. Lett., 42, 6829-6835, https://doi.org/ 10.1002/2015GL065130.

_ _ J. Tribbia, J. Anderson, and K. Raeder, 2009: Uncertainties of estimates of inertia-gravity energy in the atmosphere. Part I: Intercomparison of four analysis systems. Mon. Wea. Rev., 137, 3837-2477, https://doi.org/10.1175/2009MWR2815.1.

_ A. Kashahara, K. Terasaki, J. Tribbia, and H. Tanaka, 2015: Normal-mode function representation of global 3-D data sets: Open-access software for the atmospheric research community. Geosci. Model Dev., 8, 1169-1195, https://doi.org/10.5194/ gmd-8-1169-2015.

Zhang, C., 2005: Madden-Julian oscillation. Rev. Geophys., 43, RG2003, https://doi.org/10.1029/2004RG000158. 